\title{
ON A TWO-POINT BOUNDARY VALUE PROBLEM FOR THE 2-D NAVIER-STOKES EQUATIONS ARISING FROM CAPILLARY EFFECT
}

\author{
Bhagya Athukorallage ${ }^{1}$ And Ram IYeR ${ }^{2, *}$
}

\begin{abstract}
In this article, we consider the motion of a liquid surface between two parallel surfaces. Both surfaces are non-ideal, and hence, subject to contact angle hysteresis effect. Due to this effect, the angle of contact between a capillary surface and a solid surface takes values in a closed interval. Furthermore, the evolution of the contact angle as a function of the contact area exhibits hysteresis. We study the two-point boundary value problem in time whereby a liquid surface with one contact angle at $t=0$ is deformed to another with a different contact angle at $t=\infty$ while the volume remains constant, with the goal of determining the energy loss due to viscosity. The fluid flow is modeled by the Navier-Stokes equations, while the Young-Laplace equation models the initial and final capillary surfaces. It is well-known even for ordinary differential equations that two-point boundary value problems may not have solutions. We show existence of classical solutions that are non-unique, develop an algorithm for their computation, and prove convergence for initial and final surfaces that lie in a certain set. Finally, we compute the energy lost due to viscous friction by the central solution of the two-point boundary value problem.
\end{abstract}

Mathematics Subject Classification. 34C55, 49J40, 74S30.

Received January 1, 2019. Accepted June 25, 2019.

\section{INTRODUCTION}

Capillary action is the ability of a liquid to flow in narrow spaces without the assistance of, and in opposition to, external forces like gravity. The capillary effect is an intriguing phenomenon that is fundamental to existence of life on earth. It is due to this effect that micropores in the soil close to the surface retain water after a soaking or rainfall, allowing scattered seeds access to water. Three effects contribute to capillary action, namely, adhesion of the liquid to the walls of the confining solid; meniscus formation due to surface tension; and low Reynolds number fluid flow.

A study of the energy mechanisms involved in wetting and drying of water in soil is very important in the field of climate change, and also for determining optimal irrigation procedures for agriculture [1]. Soil pore spaces are micro capillary tubes which fill-up and dry-out as a consequence of rainfall/irrigation, and evaporation. Each cycle of wetting and drying involves a motion of the contact line and subsequent change in the curvature of the

Keywords and phrases: Capillary surfaces, contact angle hysteresis, two-point boundary value problem, 2D Navier-Stokes equation, dissipation due to viscosity.

1 Department of Mathematics and Statistics, Northern Arizona University, Flagstaff, Arizona 86001, USA.

2 Department of Mathematics and Statistics, Texas Tech University, Broadway and Boston, Lubbock, TX 79409-1042, USA.

* Corresponding author: ram.iyer@ttu.edu 
capillary interface. These two phenomena correspond to two different mechanisms for energy converted to heat (i) hysteresis that has its origins in the adhesion between liquid and solid and is quasi-static or rate-independent [4]; (ii) viscous friction that opposes the motion of neighboring fluid molecules. In earlier work [4], we studied the quasi-static hysteresis phenomenon due to capillary effect and showed that the energy required to overcome adhesion while completing a cycle is simply obtained from the area of the graph of capillary pressure versus volume of the liquid. In this article, we consider a special case of the more general theory in [4] that is amenable to computation of the energy lost due to viscosity when the capillary surface is deformed without motion of the solid-liquid contact line.

Numerical computation of the energy demand due to rate-independent hysteresis for a capillary tube is presented in [3] - for example, for a capillary tube with approximate wetting area of $0.4 \mathrm{~cm}^{2}$, and capillary surface motion of $0.006 \mathrm{~cm}$, the amount of energy converted to internal energy is $3 \mathrm{~nJ}$. In this article, we study the other phenomenon, namely, viscous friction that leads to energy dissipation in an isothermal fluid, so that one may compare numbers and get an idea of the more dominant mechanism of entropy increase (or increase in internal energy of the liquid and surroundings) in capillary effect. To enable analysis, and for clarity, we chose a simpler configuration for analysis in this article. From the results documented in Table 1, we see that for a similar wetting area and capillary line motion of $0.02 \mathrm{~cm}$, the energy converted to heat due to viscosity is $0.1 \mathrm{~nJ}$. Therefore, from the two results, we may conclude that in nano-fluidics, it is extremely important to model adhesion and the contact angle hysteresis phenomenon, as it is the dominant effect leading to increase of internal energy of the system.

As mentioned in the last paragraph, to make the problem amenable to analysis, we consider a simplified problem where a liquid and a gas are bounded between two parallel plane surfaces with a capillary surface between the liquid-gas interface (see Fig. 1). Let the contact angle, $\theta$, be the angle between the liquid surface and the solid boundary measured through the liquid. We assume solid surfaces to be non-ideal. On non-ideal surfaces, the contact angle may have a range of values due to the characteristics of the surface chemistry and topography [11]. We study the isothermal deformation of the capillary interface, where the contact angles change while the contact lines are pinned to the solid surfaces. We solve an infinite time horizon two-point boundary value problem for the initial fluid velocity distribution that deforms an initial capillary surface to another surface. In this research, we develop theoretical methods to prove existence of solutions and numerical methods to compute the solutions, and obtain values for the viscous energy dissipation.

Fluid flow between parallel plates has been of interest to many researchers. The fluid flow over an oscillating wall is studied in the well-known Stokes second problem [18]. Here, the fluid flow is a unidirectional flow in the horizontal direction. Therefore, the governing equation for the fluid flow does not contain either the pressure gradient term or the gravitational acceleration term. The Hele-Shaw fluid flow describes a flow between two horizontal plates separated by a thin gap. In [6], the authors model the Hele-Shaw flow using a version of the Stokes equation, $\nabla p=\mu \nabla^{2} \mathbf{u}$, together with a free surface boundary condition, $p=0$, that neglects the surface tension effects. Aguilar and coauthors [16] study the motion of the interface between two viscous fluids in a channel formed by two parallel solid plates. Their numerical computations reveal that advancing menisci are found for low capillary and Péclet numbers.

In [5], the authors consider a deformation of a liquid bridge formed between two horizontal, non-ideal solid surfaces. The authors model the shape of the liquid meniscus using the Young-Laplace equation [7], and neglect the effect of gravity, which results in a constant liquid pressure distribution inside the liquid bridge. They observe that the liquid bridge possesses two different equilibrium profiles with the same distance of separation of the solid surfaces. In [21], the authors estimate the thickness of a liquid film that adhere to a flat vertical plate, which moves in the vertical direction by considering a simplified version of the Navier-Stokes equation. This version of the Navier-Stokes equation results in a constant fluid film thickness along the flat plate, which can only occur in the extreme case of very low adhesion energy. In contrast to the literature, we study low Reynold's number fluid flow between two parallel plates using the two dimensional Navier-Stokes equations including gravity with adhesion of the fluid to the wall, and the capillary surface modelled using the Young-Laplace equation.

This paper is organized as follows. In Section 2, we model the capillary surface using an equation arising from the minimization of the Helmholtz energy subject to a constant volume constraint $[7,12,20]$. The energy 
of the liquid meniscus between the plates consists of capillary surface energy, wetting/adhesion energy of the solid walls bonding the liquid, and potential energy due to gravitation [10,22]. We assume invariance of the liquid surface in the $z$ direction. The height of the capillary surface, $f(x)$, is specified with respect to the depth where the liquid pressure equals the atmospheric pressure (see Fig. 1). The capillary surface, $f(x)$, defined over the domain $[0, L]$ satisfies a second-order ordinary differential equation. Specification of the contact angles at the two walls, which are related to $f^{\prime}(0)$ and $f^{\prime}(L)$, yields a two-point boundary value problem that we solve numerically using the modified simple shooting method [13]. Then, the second surface is obtained with the boundary conditions: $f(0)$ and $f(L)$, where $f(L)$ is identical to the function value from the first surface, and the volume occupied by the two surfaces are equal. In Section 3, we analyze the fluid flow under the capillary surface using the full two-dimensional Navier-Stokes and continuity equations, and solve the initial velocity distribution that takes the initial surface to the final surface. We show the existence of a classical solution to this problem. In Section 4, we solve for the initial velocity field of the liquid that takes the initial given capillary surface to a different, given surface with the volume of the liquid remaining the same. Finally, we numerically compute the viscous energy dissipation for the fluid flow.

\section{MODEL FORMULATION FOR THE CAPILlARY SURFACE}

We consider a liquid meniscus between two solid surfaces whose shape and behavior are invariant in the $z$ direction (see Fig. 1). Therefore, we may consider a unit width in the $z$ direction, and reduce the problem to a 2-dimensional one. The $x z$-plane in Figure 1 corresponds to the depth where the pressure in the liquid is equal to the atmospheric pressure. Let the liquid volume between the $x z$-plane and under the capillary surface at time $t$ be $V_{0}(t)$. The density of the liquid is $\rho$, and $\gamma_{L G}$ denotes the surface energy of the capillary surface per unit area, while $\gamma_{S L}^{1}$ and $\gamma_{S L}^{2}$ denote the adhesion energy between the liquid and the solid substrate, where the superscripts 1 and 2 respectively refer to the two parallel walls. To avoid unnecessary complications that do not yield further insight, we assume the two solid surfaces to be composed of the same material, so that the solid-liquid interface energy per unit area $\gamma_{S L}$ and the solid-gas interface energy per unit area $\gamma_{S G}$ are identical for both surfaces. Define

$$
\cos \theta_{Y}=\frac{\gamma_{S G}-\gamma_{S L}}{\gamma_{L G}}
$$

We assume that $\gamma_{S L}$ take the values in the set $\left[\gamma_{S L_{\min }}, \gamma_{S L_{\max }}\right]$. Justification for this assumption is provided in detail in $[4,9]$.

Angles of contact between the liquid surface and the solid boundaries are denoted by $\theta_{1}, \theta_{2}$, and $g$ denotes the magnitude of gravitational acceleration. Let $\bar{E}$ be the total internal energy of the capillary surface $y=f(x)$, which forms between the two unit-width plates as in Figure 1. Apriori, we assume $f \in W^{1,1}[0, L]$, the Sobolev space consisting of integrable functions with integrable derivatives, although we show later that classical solutions in $\mathrm{C}^{2}[0, L]$ exist for the uniformity in $y$ case. Considering this case is sufficient as our interest is in the computation of the scale of energy lost due to viscosity.

The shape of the capillary surface is determined by the energy functional $E$, which consists of four components: free surface energy, solid-liquid interface energy, solid-gas interface energy, and gravitational potential energy $($ see $[3])$ :

$$
\begin{aligned}
E= & \int_{0}^{L} \gamma_{L G} \sqrt{1+f^{\prime 2}(x)} \mathrm{d} x+\int_{0}^{L} \frac{1}{2} \rho g f^{2}(x) \mathrm{d} x+\gamma_{S L} f(0)+\gamma_{S L} f(L) \\
& +\gamma_{S G}\left(l_{1}-f(0)\right)+\gamma_{S G}\left(l_{2}-f(L)\right),
\end{aligned}
$$

where $l_{1}$ and $l_{2}$ are the heights of the two plates. 


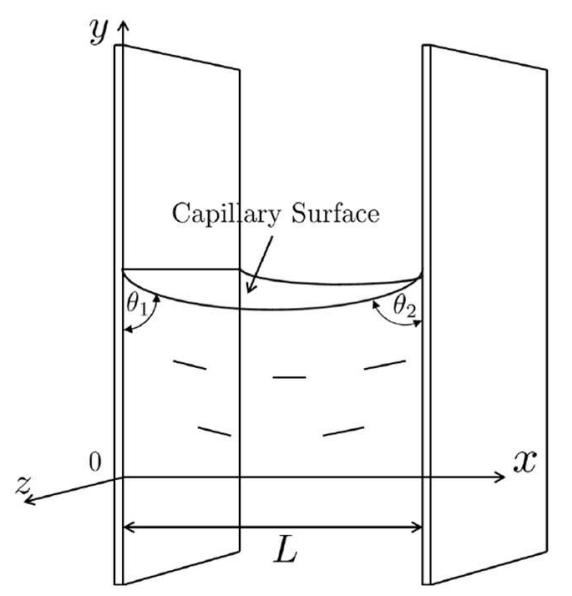

FiguRE 1. Vertical plates which are immersed in a liquid. The $y$-axis is placed along the left vertical plate, and the $x$-axis is perpendicular to the plates. Gravitational acceleration $\mathbf{g}$, acts along the $-y$ direction [2].

We assume an isothermal condition for the liquid, a unit length in the transverse direction $z$, and consider the first variation $\delta E$ of the extremal $E$ with respect to the capillary surface height $f$, with the volume

$$
V(t)=\int_{0}^{L} f(x) \mathrm{d} x
$$

which is constrained to be $V_{0}(t)$. The variations of $f$ lie in $\mathrm{H}^{2}[0, L]$. The first order necessary condition for optimality is:

$$
\delta E+\lambda(t) \delta|V(t)| \leq 0
$$

where $\lambda(t)$ is the associated Lagrange multiplier for the volume constraint. We obtain following system of equations and inequalities after a standard application of integration by parts:

$$
\begin{gathered}
\rho g f(x)-\gamma_{L G} \frac{f^{\prime \prime}(x)}{\sqrt{\left(1+f^{\prime 2}(x)\right)^{3}}}+\lambda(t)=0 \quad \text { in }[0, L], \\
\int_{0}^{L} f(x) \mathrm{d} x=V_{0}(t) \\
\left(-\left.\cos \theta_{Y}\right|_{x=0}-\frac{f^{\prime}(0)}{\sqrt{1+f^{\prime 2}(0)}}\right) \delta f(0) \leq 0 \\
\left(-\left.\cos \theta_{Y}\right|_{x=L}+\frac{f^{\prime}(L)}{\sqrt{1+f^{\prime 2}(L)}}\right) \delta f(L) \leq 0
\end{gathered}
$$


where $\delta f(x)$ is the all possible small perturbations of $f(x)$, and $\delta f(0)$ and $\delta f(L)$ are allowed to take both positive and negative values. As $f^{\prime}(0)=-\cot \theta_{1}, f^{\prime}(L)=\cot \theta_{2}$,

$$
\frac{f^{\prime}(0)}{\sqrt{1+f^{\prime 2}(0)}}=-\cos \theta_{1} ; \quad \frac{f^{\prime}(L)}{\sqrt{1+f^{\prime 2}(L)}}=\cos \theta_{2}
$$

Hence, (2.5) and (2.6) become $\forall \delta f(0), \delta f(L)$,

$$
\begin{gathered}
\left(-\left.\cos \theta_{Y}\right|_{x=0}+\cos \theta_{1}\right) \delta f(0) \leq 0 \\
\left(-\left.\cos \theta_{Y}\right|_{x=L}+\cos \theta_{2}\right) \delta f(L) \leq 0
\end{gathered}
$$

Note that the value of $\theta_{1}$ in (2.7) is related to the variation $\delta f(0)$. Now, $\cos \theta_{Y} \in\left[\cos \theta_{A}, \cos \theta_{R}\right]$, where

$$
\cos \theta_{R}=\frac{\gamma_{S G}-\gamma_{S L_{\min }}}{\gamma_{L G}} \text { and } \cos \theta_{A}=\frac{\gamma_{S G}-\gamma_{S L_{\max }}}{\gamma_{L G}}
$$

We assume the energy coefficients to take values so that $\theta_{A}$ and $\theta_{R}$ between 0 and $\frac{\pi}{2}$ with $\theta_{R}<\theta_{A}$. We study (2.7) in detail, and draw similar conclusions for (2.8).

1. $\cos \theta_{1} \in\left(\cos \theta_{A}, \cos \theta_{R}\right)$ if and only if $\forall \theta_{Y}, \cos \theta_{1}-\left.\cos \theta_{Y}\right|_{x=0}$ may be greater than, or equal to, or less than zero. Hence, by inequality $(2.7), \delta f(0)=0$.

2. $\cos \theta_{1}=\cos \theta_{A}$ if and only if $\forall \theta_{Y}, \cos \theta_{1}-\left.\cos \theta_{Y}\right|_{x=0} \leq 0$. Hence, by inequality (2.7), if $\theta_{1}=\theta_{A}, \delta f(0) \geq 0$. In other words, the capillary surface is allowed to creep up along the first wall from an extremum position if $\theta_{1}=\theta_{A}$, the advancing angle. Clearly, as the maximum value allowed for $\theta_{1}$ is $\theta_{A}$, the surface may creep up the first wall from an extremum only by maintaining $\theta_{1}$ at $\theta_{A}$.

3. $\cos \theta_{1}=\cos \theta_{R}$ if and only if $\forall \theta_{Y}, \cos \theta_{1}-\left.\cos \theta_{Y}\right|_{x=0} \geq 0$. Hence, by inequality (2.7), if $\theta_{1}=\theta_{R}, \delta f(0) \leq 0$. So, the capillary surface is allowed to creep down along the first wall from an extremum position if $\theta_{1}=\theta_{R}$, the receding angle. Just as in the previous case, the surface may creep down the first wall from an extremum only by maintaining $\theta_{1}$ at $\theta_{R}$.

The above analysis shows that the motion of the contact line of the capillary surface on either surfaces is subject to hysteresis. In this article, we study case (1) above by deforming a capillary surface without moving the contact line.

Elimination of the Lagrange multiplier. The Lagrange multiplier $\lambda(t)$ may be eliminated by integrating (2.3) as $\int_{0}^{L} f(x) \mathrm{d} x=V_{0}(t)$. Finally, the equation for the capillary surface is: $\forall x \in[0, L]$,

$$
\rho g f(x)-\gamma_{L G} \frac{f^{\prime \prime}(x)}{\sqrt{\left(1+f^{\prime 2}(x)\right)^{3}}}+\frac{\gamma_{L G}\left(\cos \theta_{1}+\cos \theta_{2}\right)-\rho g V_{0}}{L}=0 .
$$

Further, we see that by letting $f(x)=v(x)-\frac{\lambda(t)}{\rho g},(2.3)$ may be simplified to

$$
\frac{v^{\prime \prime}}{\left(1+v^{\prime 2}\right)^{3 / 2}}=\kappa v \quad \text { in }[0, L]
$$

with the boundary conditions $v^{\prime}(0)=-\cot \left(\theta_{1}\right)$ and $v^{\prime}(L)=\cot \left(\theta_{2}\right)$. 


\section{AN INFINITE TIME HORIZON TWO-POINT BOUNDARY VALUE PROBLEM}

We consider the motion of the left vertical plate in Figure 1 in the positive $y$ direction. In this section, we solve the initial velocity field of the liquid that takes the initial given capillary surface to a different, given surface with the volume of the liquid remaining the same.

Let $f(x, t)$ be the capillary surface at time $t$, and let the initial and final capillary surfaces be $f_{i}=f(x, 0)$ and $f_{f}=f(x, \infty)$ (see Fig. 2) that satisfy (2.3) and (2.4). The initial domain $D(0):=[0, L] \times\left[0, f_{i}\right]$ and the final domain $D(\infty):=[0, L] \times\left[0, f_{f}\right]$ such that $D(0)$ and $D(\infty)$ posses equal areas. We consider a Newtonian, incompressible fluid with velocity $\mathbf{u}=(u(x, y, t), v(x, y, t))$, where $u$ and $v$ denote the fluid velocity components in the $x$ and $y$ directions. The fluid flow is analyzed using the Navier-Stokes and the continuity equations [8]:

$$
\rho\left(\frac{\partial \mathbf{u}}{\partial t}+\mathbf{u} \cdot \nabla \mathbf{u}\right)=\rho \mathbf{g}-\nabla p+\mu \nabla^{2} \mathbf{u} \quad \text { and } \quad \nabla \cdot \mathbf{u}=0
$$

together with the boundary conditions $u(0, y, t)=u(L, y, t)=0$. The problem is to solve the initial velocity of the fluid that results in transforming the domain $D(0)$ to $D(\infty)$.

Let $(x, y)$ be Eulerian coordinates in the domain $D(t)$ defined by $0 \leq x \leq L$ and $0 \leq y \leq f(x, t)$ at time $t$, and let $\left(x_{0}, y_{0}\right)$ be the particles in the initial domain $D(0)$.

Then:

$$
\frac{\mathrm{d}(x, y)}{\mathrm{d} t}=(u(x, y, t), v(x, y, t)) \quad \text { with } \quad(x, y)(0)=\left(x_{0}, y_{0}\right)
$$

Therefore:

$$
\begin{aligned}
(x, y)(t) & =\left(x_{0}, y_{0}\right)+\int_{0}^{t}\left(U\left(x_{0}, y_{0}, \tau\right), V\left(x_{0}, y_{0}, \tau\right)\right) \mathrm{d} \tau \\
& =\left(X\left(x_{0}, y_{0}, t\right), Y(\xi, \eta, t)\right)
\end{aligned}
$$

where $U\left(x_{0}, y_{0}, t\right)=u(x, y, t)=u\left(X\left(x_{0}, y_{0}, t\right), Y\left(x_{0}, y_{0}, t\right)\right)$ and similarly for $v$ and $V$. Observe that in (3.1), $u(x, y, t)$ and $v(x, y, t)$ are the velocity components in the $x$ and $y$ directions, respectively. We use the integral version of the corresponding kinematic free-surface boundary condition

$$
\frac{\partial f(x, t)}{\partial t}+u \frac{\partial f(x, t)}{\partial x}=v
$$

Here, $f(x, t)$ is the capillary surface at time $t$, and hence integrating (3.2) with respect to the $t$ variable yields,

$$
f(x, t)=f_{i}(x)+\int_{0}^{t}\left(v(x, y(x, s), s)-\frac{\partial f(x, s)}{\partial x} u(x, s)\right) \mathrm{d} s .
$$

As the liquid has zero velocity in the $x$ direction at $x=0$ and $x=L$, we consider, $u(x, y, t)=u_{0}(t) \sin \left(\frac{\pi x}{L}\right) \phi(y)$. Then, the continuity equation [8] yields:

$$
v(x, y, t)=A(x, t)-\frac{\pi}{L} u_{0}(t) \cos \left(\frac{\pi x}{L}\right) \int_{0}^{y} \phi(\tau) \mathrm{d} \tau .
$$




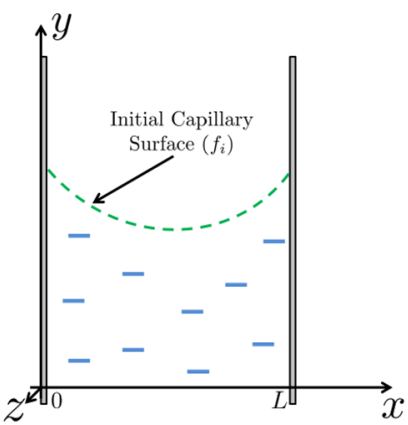

(a)

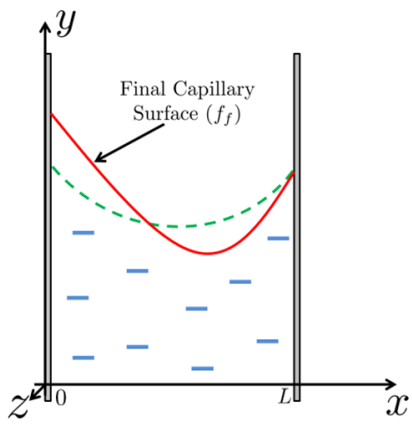

(b)

Figure 2. Schematic drawing of (a) initial $\left(f_{i}\right)$ and (b) final $\left(f_{f}\right)$ capillary surfaces.

Assume that the liquid has dynamic viscosity $\mu$ and density $\rho$. From the $x$-component of the 2 -dimensional Navier-Stokes equation [8]:

$$
\rho\left(\frac{\partial u}{\partial t}+u \frac{\partial u}{\partial x}+v \frac{\partial u}{\partial y}\right)=-\frac{\partial p}{\partial x}+\mu\left(\frac{\partial^{2} u}{\partial^{2} x}+\frac{\partial^{2} u}{\partial^{2} y}\right)
$$

it follows that

$$
\begin{aligned}
& u_{0}^{\prime}(t) \phi(y) \sin \left(\frac{\pi x}{L}\right)+\left(A(x, t)-\frac{\pi}{L} u_{0}(t) \cos \left(\frac{\pi x}{L}\right) \int_{0}^{y} \phi(\tau) \mathrm{d} \tau\right) u_{0}(t) \sin \left(\frac{\pi x}{L}\right) \phi^{\prime}(y) \\
& +\frac{\pi}{2 L} u_{0}^{2}(t) \phi^{2}(y) \sin \left(\frac{2 \pi x}{L}\right)=-\frac{1}{\rho} \frac{\partial p}{\partial x}+\frac{\mu}{\rho}\left(-u_{0}(t) \phi(y) \frac{\pi^{2}}{L^{2}} \sin \left(\frac{\pi x}{L}\right)+u_{0}(t) \sin \left(\frac{\pi x}{L}\right) \phi^{\prime \prime}(y)\right) .
\end{aligned}
$$

In general, $\phi(y)=\sum_{k=0}^{\infty} a_{k} y^{k}$, but we notice that $\phi(y)=a_{0}$ leads to one class of solutions. For $\phi(y)=a_{0}=1$, we equate $\sin \left(\frac{\pi x}{L}\right)$ terms to zero in (3.6) and get the following equation for $u_{0}(t): u_{0}(t)=e^{-\frac{\mu}{\rho} \frac{\pi^{2}}{L^{2}} t} \bar{u}_{0}$. Here both $a_{0}$ and $\bar{u}_{0}$ are constants. Furthermore, using (3.6) we also get,

$$
\frac{\rho}{2} u_{0}^{2}(t) \sin \left(\frac{2 \pi x}{L}\right) \frac{\pi}{L}=-\frac{\partial p}{\partial x} .
$$

Therefore, the pressure inside the fluid is

$$
p(x, y, t)=\frac{\rho}{4} u_{0}^{2}(t) \cos \left(\frac{2 \pi x}{L}\right)+\bar{p}(y, t) .
$$

The fluid velocity in the $y$ direction, $v(x, y, t)$ is

$$
v(x, y, t)=A(x, t)-\frac{\pi}{L} u_{0}(t) \cos \left(\frac{\pi x}{L}\right) y .
$$

Next, we apply techniques of Fourier series to the $y$-component of the NS equation (see [14] for the theory of Fourier series applied to PDEs). Assuming $A(x, t)$ has the Fourier series

$$
A(x, t)=\frac{\alpha_{0}(t)}{2}+\sum_{k=1}^{\infty} \alpha_{k}(t) \cos \left(\frac{k \pi x}{L}\right)+\sum_{k=1}^{\infty} \beta_{k}(t) \sin \left(\frac{k \pi x}{L}\right)
$$


over the interval $[-L, L]$ where for each $t$, it is also assumed that $A(x, t)=A(-x, t)$. As $A(x, t)$ is an even function, $\beta_{k}(t)=0$ for $k \geq 1$. Next, we look at the $y$ component of the Navier-Stokes equation:

$$
\rho\left(\frac{\partial v}{\partial t}+u \frac{\partial v}{\partial x}+v \frac{\partial v}{\partial y}\right)=-\rho g-\frac{\partial p}{\partial y}+\mu\left(\frac{\partial^{2} v}{\partial x^{2}}+\frac{\partial^{2} v}{\partial y^{2}}\right)
$$

Here, $u(x, y, t)=u_{0}(t) \sin \left(\frac{\pi x}{L}\right), \rho$ and $\mu$ are the density and viscosity of the liquid. Gravitational acceleration $g$ acts in the $-y$ direction.

Equations (3.8), (3.9), and (3.10) yield:

$$
\begin{aligned}
& \frac{\alpha_{0}^{\prime}(t)}{2}+\sum_{k=1}^{\infty} \alpha_{k}^{\prime}(t) \cos \left(\frac{k \pi x}{L}\right)-\frac{\pi}{L} u_{0}^{\prime}(t) \cos \left(\frac{\pi x}{L}\right) y+\frac{\pi^{2}}{L^{2}} u_{0}^{2}(t) \sin ^{2}\left(\frac{\pi x}{L}\right) y \\
& -\frac{\pi}{L} u_{0}(t) \sin \left(\frac{\pi x}{L}\right)\left(\sum_{k=1}^{\infty} k \alpha_{k}(t) \sin \left(\frac{k \pi x}{L}\right)\right)+\frac{\pi^{2}}{L^{2}} u_{0}^{2}(t) \cos ^{2}\left(\frac{\pi x}{L}\right) y \\
& -\frac{\pi}{L} u_{0}(t) \cos \left(\frac{\pi x}{L}\right)\left(\frac{\alpha_{0}(t)}{2}+\sum_{k=1}^{\infty} \alpha_{k}(t) \cos \left(\frac{k \pi x}{L}\right)\right) \\
& =-g-\frac{1}{\rho} \frac{\partial \bar{p}}{\partial y}+\frac{\mu \pi^{2}}{\rho L^{2}}\left(\frac{\pi}{L} u_{0}(t) \cos \left(\frac{\pi x}{L}\right) y-\sum_{k=1}^{\infty} k^{2} \alpha_{k}(t) \cos \left(\frac{k \pi x}{L}\right)\right) .
\end{aligned}
$$

As $\bar{p}$ is only a function of $y$ and $t$, the second term on the right-hand side cannot be a function of terms of the type $\sin \left(\frac{k \pi x}{L}\right)$ or $\cos \left(\frac{k \pi x}{L}\right)$ for $k \geq 1$. For $k=0$, we have:

$$
\frac{\alpha_{0}^{\prime}(t)}{2}-\frac{\pi}{L} u_{0}(t) \alpha_{1}(t)+\frac{\pi^{2}}{L^{2}} u_{0}^{2}(t) y=-g-\frac{1}{\rho} \frac{\partial \bar{p}}{\partial y}
$$

Observe in (3.12), there are terms that depend on $t$ and $y$, and terms that depend only on $t$. If $\frac{\alpha_{0}^{\prime}(t)}{2}=$ $\left(-g+\right.$ other terms), we see that $\int_{0}^{\infty} \alpha_{0}(s) \mathrm{d} s$ is unbounded. This cannot happen for the following reason. Let the initial and the final capillary surfaces be $f_{i}(x)$ and $f_{f}(x)$, respectively. We can relate the $f_{i}(x), f_{f}(x)$, and $v(x, y, t)$ according to integral version of the kinematic boundary condition (see (3.2)):

$$
f_{f}(x)-f_{i}(x)=\int_{0}^{\infty}\left(v(x, y(x, t), t)-\frac{\partial f(x, t)}{\partial x} u(x, t)\right) \mathrm{d} t .
$$

Thus, substituting (3.8) into (3.13) and rearranging the terms, one obtains

$$
\begin{aligned}
f_{f}(x)-f_{i}(x)= & -\frac{\pi}{L} \cos \left(\frac{\pi x}{L}\right) \int_{0}^{\infty} y(x, t) u_{0}(t) \mathrm{d} t-\int_{0}^{\infty} \frac{\partial f(x, t)}{\partial x} u(x, t) \mathrm{d} t \\
& +\int_{0}^{\infty}\left(\frac{\alpha_{0}(t)}{2}+\sum_{k=1}^{\infty} \alpha_{k}(t) \cos \left(\frac{k \pi x}{L}\right)\right) \mathrm{d} t .
\end{aligned}
$$

We see that a solution does not exist if $\int_{0}^{\infty} \alpha_{0}(s) \mathrm{d} s$ is unbounded. Note that

$$
\int_{0}^{\infty} \sum_{k=1}^{\infty} \alpha_{k}(t) \cos \left(\frac{k \pi x}{L}\right) \mathrm{d} t=\sum_{k=1}^{\infty}\left(\int_{0}^{\infty} \alpha_{k}(t) \mathrm{d} t\right) \cos \left(\frac{k \pi x}{L}\right)
$$


is a property that we desire for the solution and is guaranteed if the sequence of functions $\left\{\alpha_{i}\right\}$ are collectively in $l^{2}\left(L^{2}[0, \infty)\right)$.

If, for some function $\xi$, the functions $\alpha_{0}, \alpha_{1}, u_{0}$ and $\bar{p}$ satisfy

$$
\frac{\alpha_{0}^{\prime}(t)}{2}-\frac{\pi}{L} u_{0}(t) \alpha_{1}(t)=\xi(t) \quad \text { and } \quad \frac{\pi^{2}}{L^{2}} u_{0}^{2}(t) y+g+\frac{1}{\rho} \frac{\partial \bar{p}}{\partial y}=-\xi(t)
$$

then they automatically satisfy (3.12). Here, we have separated the terms with $y$ dependence from those that do not have $y$ dependence, and $g$ is decoupled from $\alpha_{0}^{\prime}$ due to the reason we just described. Thus, different choices of $\xi$ lead to different solutions. If $\xi(t)=0$ then there does not exist the solution, for the following reason. By equating the coefficients of the $\cos \left(\frac{k \pi x}{L}\right)$ for $k \geq 1$ in (3.11), we obtain the system of equations (where $\varphi(t)=\frac{\pi u_{0}(t)}{2 L}$ and $\left.\eta=\frac{\mu \pi^{2}}{\rho L^{2}}\right)$ :

$$
\left[\begin{array}{c}
\alpha_{0}^{\prime}(t) \\
\alpha_{1}^{\prime}(t) \\
\alpha_{2}^{\prime}(t) \\
\alpha_{3}^{\prime}(t) \\
\alpha_{4}^{\prime}(t) \\
\alpha_{5}^{\prime}(t) \\
\vdots
\end{array}\right]=\left[\begin{array}{cc|ccccc}
0 & 4 \varphi(t) & 0 & 0 & 0 & 0 & \cdots \\
\varphi(t) & -\eta & 3 \varphi(t) & 0 & 0 & 0 & \cdots \\
\hline 0 & 0 & -4 \eta & 4 \varphi(t) & 0 & 0 & \cdots \\
0 & 0 & -\varphi(t) & -9 \eta & 5 \varphi(t) & 0 & \cdots \\
0 & 0 & 0 & -2 \varphi(t) & -16 \eta & 6 \varphi(t) & \cdots \\
0 & 0 & 0 & 0 & -3 \varphi(t) & -25 \eta & \cdots \\
\vdots & \vdots & \vdots & \vdots & \vdots & \vdots & \ddots
\end{array}\right]\left[\begin{array}{c}
\alpha_{0}(t) \\
\alpha_{1}(t) \\
\alpha_{2}(t) \\
\alpha_{3}(t) \\
\alpha_{4}(t) \\
\alpha_{5}(t) \\
\vdots
\end{array}\right]
$$

The submatrix $\left(\begin{array}{cc}0 & 4 \varphi(t) \\ \varphi(t) & -\eta\end{array}\right)$ leads to unstable dynamics for $\alpha_{0}(t)$ and $\alpha_{1}(t)$ with $\alpha_{2}(t)=0$ (observe that for each $t \geq 0$ one of the eigenvalues of this matrix is a positive real number $)$. For stabilizing $\left(\alpha_{0}, \alpha_{1}\right), \alpha_{2}$ must be a function of $\alpha_{0}$ and $\alpha_{1}$. However, in (3.15), $\alpha_{2}$ depends on neither $\alpha_{0}$ nor $\alpha_{1}$. Therefore, $\left(\alpha_{0}, \alpha_{1}\right)$ are unbounded functions of time irrespective of the other coefficients. Hence, $\xi(\cdot)$ must stabilize the system

$$
\left(\begin{array}{c}
\alpha_{0}^{\prime}(t) \\
\alpha_{1}^{\prime}(t)
\end{array}\right)=\left(\begin{array}{cc}
0 & 4 \varphi(t) \\
\varphi(t) & -\eta
\end{array}\right)\left(\begin{array}{c}
\alpha_{0}(t) \\
\alpha_{1}(t)
\end{array}\right)+\left(\begin{array}{c}
\xi(t) \\
0
\end{array}\right)
$$

in order to solutions to exist. It is clear that $\xi(t)$ must be of the form $\xi(t)=-\epsilon \alpha_{0}(t)+\tilde{\xi}(t)$ for some function $\tilde{\xi}(t)$ and $\epsilon>0$, as otherwise, the linear system is unstable. Setting $\tilde{\xi}(t)=0$, we obtain a $\epsilon$-central solution, that is, a solution dependent on $\epsilon$ which we call "central", because other stable solutions are perturbations of this solution. Hence, for our numerical computations, we modify the system in (3.15) with $\alpha_{0}^{\prime}(t)=-\epsilon \alpha_{0}(t)+4 \varphi(t) \alpha_{1}(t)$. The modified system is:

$$
\left[\begin{array}{c}
\alpha_{0}^{\prime}(t) \\
\alpha_{1}^{\prime}(t) \\
\alpha_{2}^{\prime}(t) \\
\alpha_{3}^{\prime}(t) \\
\alpha_{4}^{\prime}(t) \\
\alpha_{5}^{\prime}(t) \\
\vdots
\end{array}\right]=\left[\begin{array}{ccccccc}
-\epsilon & 4 \varphi(t) & 0 & 0 & 0 & 0 & \cdots \\
\varphi(t) & -\eta & 3 \varphi(t) & 0 & 0 & 0 & \ldots \\
0 & 0 & -4 \eta & 4 \varphi(t) & 0 & 0 & \cdots \\
0 & 0 & -\varphi(t) & -9 \eta & 5 \varphi(t) & 0 & \ldots \\
0 & 0 & 0 & -2 \varphi(t) & -16 \eta & 6 \varphi(t) & \cdots \\
0 & 0 & 0 & 0 & -3 \varphi(t) & -25 \eta & \ldots \\
\vdots & \vdots & \vdots & \vdots & \vdots & \vdots & \ddots
\end{array}\right]\left[\begin{array}{c}
\alpha_{0}(t) \\
\alpha_{1}(t) \\
\alpha_{2}(t) \\
\alpha_{3}(t) \\
\alpha_{4}(t) \\
\alpha_{5}(t) \\
\vdots
\end{array}\right]
$$

Next, we analyze the solution of the system (3.17). 
Lemma 3.1. Consider the submatrix that is defined in the system:

$$
\left(\begin{array}{c}
\alpha_{0}^{\prime}(t) \\
\alpha_{1}^{\prime}(t)
\end{array}\right)=\left(\begin{array}{cc}
-\epsilon & 4 \varphi(t) \\
\varphi(t) & -\eta
\end{array}\right)\left(\begin{array}{c}
\alpha_{0}(t) \\
\alpha_{1}(t)
\end{array}\right)
$$

and let $\widehat{\alpha}_{0}(t)=\left(\alpha_{0}(t), \alpha_{1}(t)\right)$. Let $\epsilon=\eta$. Then $\exists \widetilde{k}_{1}, \widetilde{k}_{2}>0$ such that

(i) $\left\|\widehat{\alpha}_{0}(t)\right\|_{2} \leq \widetilde{k}_{1} e^{-\widetilde{k}_{2} t}\left\|\widehat{\alpha}_{0}(0)\right\|_{2}$

(ii) Furthermore, if the sequence $\widehat{\alpha}_{0}(0)=\left(\alpha_{0}(0), \alpha_{1}(0)\right) \in l^{2}$ then $\forall t>0 \widehat{\alpha}_{0}(t) \in l^{2}$.

Proof. See Appendix A.1.

Lemma 3.2. Let $\widehat{\alpha}_{N}(t)=\left(\alpha_{2}(t), \alpha_{3}(t), \ldots, \alpha_{N}(t)\right)$. Then $\exists k_{1}, k_{2}>0$ that do not depend on $N$ such that:

(i) $\left\|\widehat{\alpha}_{N}(t)\right\|_{2} \leq k_{1} e^{-k_{2} t}\left\|\widehat{\alpha}_{N}(0)\right\|_{2}$

(ii) Furthermore, if the sequence $\widehat{\alpha}(0)=\left(\alpha_{2}(0), \alpha_{3}(0), \ldots\right) \in l^{2}$ then $\forall t>0 \widehat{\alpha}(t) \in l^{2}$.

Proof. See Appendix A.2.

Lemma 3.3. Consider the system:

$$
\left(\begin{array}{c}
\alpha_{0}^{\prime}(t) \\
\alpha_{1}^{\prime}(t)
\end{array}\right)=\left(\begin{array}{cc}
-\epsilon & 4 \varphi(t) \\
\varphi(t) & -\eta
\end{array}\right)\left(\begin{array}{c}
\alpha_{0}(t) \\
\alpha_{1}(t)
\end{array}\right)+\left(\begin{array}{c}
0 \\
3 \varphi(t)
\end{array}\right) \alpha_{2}(t)
$$

Then Lemmas 3.1 and 3.2 yield

$$
\left\|\widehat{\alpha}_{0}(t)\right\|_{2} \leq \widetilde{k}_{1} e^{-\widetilde{k}_{2} t}\left\|\widehat{\alpha}_{0}(0)\right\|_{2}+\frac{\widetilde{k}_{1} k_{1}}{k_{2}-\widetilde{k}_{2}}\left(e^{-\widetilde{k}_{2} t}-e^{-k_{2} t}\right)\left\|\widehat{\alpha}_{N}(0)\right\| .
$$

Furthermore, if $\widehat{\alpha}(t)=\left(\alpha_{0}(t), \alpha_{1}(t), \alpha_{2}(t), \ldots,\right)$, one may obtain the following result:

$$
\|\widehat{\alpha}(t)\|_{2} \leq K_{1} e^{-K_{2} t}\|\widehat{\alpha}(0)\|_{2} .
$$

Here, $K_{1}=\max \left\{k_{1}, \widetilde{k}_{1}\right\}$ and $K_{2}=\min \left\{k_{2}, \widetilde{k}_{2}\right\}$.

Proof. See Appendix A.3.

Lemma 3.4. Let $\theta_{k}(t):=k \alpha_{k}(t)$ and $\widehat{\theta}_{N}(t)=\left(\theta_{2}(t), \theta_{3}(t), \ldots, \theta_{N}(t)\right)$. Then, $\exists a_{1}, a_{2}>0$ that do not depend on $N$ such that:

(i) $\left\|\widehat{\theta}_{N}(t)\right\|_{2} \leq a_{1} e^{-a_{2} t}\left\|\widehat{\theta}_{N}(0)\right\|_{2}$

(ii) Furthermore, if the sequence $\widehat{\theta}(0)=\left(\theta_{2}(0), \theta_{3}(0), \ldots\right) \in l^{2}$ then $\forall t>0 \hat{\theta}(t) \in l^{2}$.

Lemma 3.5. Let $\gamma_{k}(t):=k^{2} \alpha_{k}(t)$ and $\widehat{\gamma}_{N}(t)=\left(\gamma_{2}(t), \gamma_{3}(t), \ldots, \gamma_{N}(t)\right)$. Then, $\exists m_{1}, m_{2}>0$ that do not depend on $N$ such that:

(i) $\left\|\widehat{\gamma}_{N}(t)\right\|_{2} \leq m_{1} e^{-m_{2} t}\left\|\widehat{\gamma}_{N}(0)\right\|_{2}$

(ii) Furthermore, if the sequence $\widehat{\gamma}(0)=\left(\gamma_{0}(0), \gamma_{1}(0), \gamma_{2}(0), \gamma_{3}(0), \ldots\right) \in l^{2}$ then $\forall t>0 \widehat{\gamma}(t) \in l^{2}$.

Lemma 3.6. Let $\beta_{k}(t):=k^{3} \alpha_{k}(t)$ and $\widehat{\beta}_{N}(t)=\left(\beta_{2}(t), \beta_{3}(t), \ldots, \beta_{N}(t)\right)$. Then, $\exists n_{1}, n_{2}>0$ that do not depend on $N$ such that:

(i) $\left\|\widehat{\beta}_{N}(t)\right\|_{2} \leq n_{1} e^{-n_{2} t}\left\|\widehat{\beta}_{N}(0)\right\|_{2}$

(ii) Furthermore, if the sequence $\widehat{\beta}(0)=\left(\beta_{2}(0), \beta_{3}(0), \ldots\right) \in l^{2}$ then $\forall t>0 \widehat{\beta}(t) \in l^{2}$.

Note: We omit the proofs of Lemmas 3.4, 3.5, and 3.6, which are similar to that of Lemma 3.7. 
Lemma 3.7. Let $\lambda_{k}(t):=k^{4} \alpha_{k}(t)$ and $\widehat{\lambda}_{N}(t)=\left(\lambda_{2}(t), \lambda_{3}(t), \ldots, \lambda_{N}(t)\right)$. Then, $\exists p_{1}, p_{2}>0$ that do not depend on $N$ such that:

(i) $\left\|\widehat{\lambda}_{N}(t)\right\|_{2} \leq p_{1} e^{-p_{2} t}\left\|\widehat{\lambda}_{N}(0)\right\|_{2}$

(ii) Furthermore, if the sequence $\widehat{\lambda}(0)=\left(\lambda_{0}(0), \lambda_{1}(0), \lambda_{2}(0), \lambda_{3}(0), \ldots\right) \in l^{2}$ then $\forall t>0 \widehat{\lambda}(t) \in l^{2}$.

Proof. See Appendix A.4.

Moreover, if $\widehat{\alpha}(t)=\left(\alpha_{2}(t), \alpha_{3}(t), \ldots\right)$ then the relations:

$\|\widehat{\alpha}(t)\|_{2} \leq\|\widehat{\theta}(t)\|_{2} \leq\|\widehat{\gamma}(t)\|_{2} \leq\|\widehat{\beta}(t)\|_{2} \leq\|\widehat{\lambda}(t)\|_{2} \leq p_{1} e^{-p_{2} t}\|\widehat{\lambda}(0)\|_{2}$, yield

$$
\int_{0}^{\infty}\|\widehat{\alpha}(t)\|_{2} \mathrm{~d} t \leq \int_{0}^{\infty}\|\widehat{\theta}(t)\|_{2} \mathrm{~d} t \leq \int_{0}^{\infty}\|\widehat{\gamma}(t)\|_{2} \mathrm{~d} t \leq \int_{0}^{\infty}\|\widehat{\beta}(t)\|_{2} \mathrm{~d} t \leq \int_{0}^{\infty}\|\widehat{\lambda}(t)\|_{2} \mathrm{~d} t \leq \frac{p_{1}}{p_{2}}\|\widehat{\lambda}(0)\|_{2}
$$

Recall that $\widehat{\lambda}(0)=\left\{k^{4} \alpha_{k}(0)\right\}_{k=0}^{\infty}$.

Lemma 3.8. Let $\widehat{\lambda}(0) \in l^{2}$ and $\bar{u}_{0} \in \mathbb{R}$. Define $\eta=\frac{\mu \pi^{2}}{\rho L^{2}}, \omega=\frac{\pi \bar{u}_{0}}{L}$, and the function $\widetilde{v}(x, t)=|A(x, t)|+$ $\frac{\pi}{L}\left|u_{0}(t)\right||f(x, t)|$, where $f(x, t)$ satisfies $(3.3)$ with $(x, t) \in[0, L] \times[0, \infty)$. There exist $\Omega$ and $\widehat{\Omega}>0$ such that

$$
\|\widetilde{v}\|_{W^{2}} \leq \begin{cases}\frac{K_{1}^{2}\|\widehat{\alpha}(0)\|_{2}^{2}}{2 K_{2}}\left(1+\omega^{2}\left(\widehat{\Omega}+\frac{1}{\eta^{2}}\right)\right)+L\|\sigma\|^{2}\left(\Omega+\frac{1}{\eta}\right) & \text { if } \omega \geq \eta \\ \frac{K_{1}^{2}\|\widehat{\alpha}(0)\|_{2}^{2}}{2 K_{2}}\left(1+\frac{\omega^{2}}{4 \eta^{2}}\right)+\frac{L\|\sigma\|^{2}}{\eta} & \text { if } \omega<\eta .\end{cases}
$$

Hence, $\widetilde{v} \in W^{2}([0, L] \times(0, \infty))$.

Proof. See Appendix B.1.

Recall that the initial domain $D(0)=[0, L] \times\left[0, f_{i}\right]$ and the domain $D(t)=[0, L] \times[0, f(x, t)]$ at time $t$.

Theorem 3.9. $\forall\left(x_{0}, y_{0}\right) \in D(0), \lim _{t \rightarrow \infty}\left(U\left(x_{0}, y_{0}, t\right), V\left(x_{0}, y_{0}, t\right)\right)=0$.

Proof. Observe that $\forall(x, t) \in([0, L] \times(0, \infty))$, we have

$$
\left|V\left(x_{0}, y_{0}, t\right)\right|=\left|A(x, t)-\frac{\pi}{L} u_{0}(t) \cos \left(\frac{\pi x}{L}\right) y\right| \leq|A(x, t)|+\frac{\pi}{L}\left|u_{0}(t)\right||f(x, t)|=|\widetilde{v}(x, t)|
$$

and $\left|U\left(x_{0}, y_{0}, t\right)\right|=\left|\bar{u}_{0} e^{-\eta t} \sin \left(\frac{\pi x}{L}\right)\right| \leq\left|\bar{u}_{0}\right| e^{-\eta t}$. By Lemma 3.8,

$$
\int_{0}^{\infty} \int_{0}^{L}|\widetilde{v}(x, t)|^{2} \mathrm{~d} x \mathrm{~d} t<\infty
$$

That is, $\forall x \in[0, L], \lim _{t \rightarrow \infty}\|\widetilde{v}(x, t)\|=0$. We also get $\int_{0}^{\infty} \int_{0}^{L}\left|U\left(x_{0}, y_{0}, t\right)\right|^{2} \mathrm{~d} x \mathrm{~d} t \leq \frac{\left|\bar{u}_{0}\right| L}{\eta}<\infty$. Hence, $\forall\left(x_{0}, y_{0}\right) \in D(0), \lim _{t \rightarrow \infty}\left\|\left(U\left(x_{0}, y_{0}, t\right), V\left(x_{0}, y_{0}, t\right)\right)\right\|=0$.

The above theorem motivates the definition of the set of all possible domains that may be achieved at $t=\infty$. As the only part of the domains that changes with $t$ is the boundary specified by $f(\cdot, t)$, we can characterize the set of domains reachable from an initial one $f_{i}=f(\cdot, 0)$ through $\lim _{t \rightarrow \infty} f(\cdot, t)$. 
Definition 3.10. Define the reachable set

$$
\mathcal{R}\left(f_{i}\right)=\left\{\lim _{t \rightarrow \infty} f(\cdot, t): f_{i} \text { satisfies }(2.3) \text { and }(2.4) ; \widehat{\lambda}(0) \in l^{2} \text { and } \bar{u}_{0} \in \mathbb{R} ; f \text { satisfies }(3.3)\right\} .
$$

Note that an element of $\mathcal{R}\left(f_{i}\right)$ is a function defined on the domain $[0, L]$.

Theorem 3.11. Let $f_{i} \geq 0$ such that $f_{i} \in L^{2}([0, L])$ and $f_{i}$ satisfies (2.3)-(2.4). Further suppose that $f(x, t)$ satisfies (3.3). If $h \in \mathcal{R}\left(f_{i}\right)$, then $\exists \bar{u}_{0} \in \mathbb{R}$ and $\alpha_{i}(0), i \in \mathbb{N}$ with $\widehat{\lambda}(0) \in l^{2}$, such that $h(x)=\lim _{t \rightarrow \infty} f(x, t)$. Furthermore, $\widehat{\alpha} \in l^{2}\left(\mathrm{C}^{1}(0, \infty)\right)$. Therefore, if a solution to the infinite time horizon two-point boundary value problem exists, then a classical solution exists.

Proof. If $h \in R\left(f_{i}\right)$, then $h(x)=\lim _{t \rightarrow \infty} f(x, t)$ for some function $f(x, t)$ that satisfies (3.3) with $f(x, 0)$ satisfying (2.3) and (2.4), with $\bar{u}_{0} \in \mathbb{R}$ and $\widehat{\lambda}(0) \in l^{2}$. By Lemma 3.8, $\widetilde{v}(x, t) \in W^{2}([0, L] \times(0, \infty))$. By Theorem 3.9, $\lim _{t \rightarrow \infty} V\left(x_{0}, y_{0}, t\right)=0$ for $\left(x_{0}, y_{0}\right) \in D(0)$. For $\alpha \in \mathrm{C}^{1}(0, \infty)$, the norm that we employ is $\|\alpha(\cdot)\|_{\mathrm{C}^{1}}:=\sum_{i=0}^{1} \sup _{t \geq 0}\left|\alpha^{(i)}(t)\right|$. Hence,

$$
\sum_{k=0}^{\infty}\left\|\alpha_{k}(\cdot)\right\|_{\mathrm{C}^{1}}^{2}=\sum_{k=0}^{\infty}\left[\sup _{t \geq}\left|\alpha_{k}(t)\right|+\sup _{t \geq 0}\left|\alpha_{k}^{\prime}(t)\right|\right]^{2} \leq \sum_{k=0}^{\infty} \sup _{t \geq 0}\left|\alpha_{k}(t)\right|^{2}+2 \sum_{k=0}^{\infty} \sup _{t \geq 0}\left|\alpha_{k}^{\prime}(t)\right|^{2} .
$$

By using Lemma 3.3 and (A.12) (see Appendix A.1), we have

$$
\sum_{k=0}^{\infty}\left\|\alpha_{k}(\cdot)\right\|_{\mathrm{C}^{1}}^{2} \leq 2 K_{1}^{2}\|\widehat{\alpha}(0)\|_{2}^{2}+2\left[20\left(\frac{\pi \bar{u}_{0}}{2 L}\right)^{2}+6 \eta^{2}\right] m_{1}^{2}\|\widehat{\gamma}(0)\|_{2}^{2}<\infty .
$$

Hence, $\widehat{\alpha} \in l^{2}\left(\mathrm{C}^{1}(0, \infty)\right)$, and hence, $u(x, y, t), v(x, y, t)$ are continuously differentiable functions of time $t$. By Lemmas B.1, B.2 (see Appendix), $u_{x}, u_{y}, v_{x}, v_{y}, u_{x x}, u_{y y}, v_{x x}$, and $v_{y y}$ are continuously differentiable functions of time $t$. This concludes the last assertion of the theorem.

\section{NumericAl RESUlts AND DISCUSSION}

In this section, we compute the initial and the final capillary surfaces that satisfy both the equations (2.3) and (2.4). Then, we solve for the initial velocity field of the liquid that takes the initial given capillary surface $\left(f_{i}(x)\right)$ to a different, given surface $\left(f_{f}(x)\right)$ with the volume of the liquid remaining the same. Finally, the viscous energy dissipation is calculated for the fluid flow that results due to the deformation of the surfaces.

\subsection{Numerical solutions for the initial and final capillary surface}

Let the capillary surface height be $f(x)$ over the interval $[0, L]$. Recall that $f_{i}(x)$ and $f_{f}(x)$ denote the initial and final capillary surfaces, respectively. We numerically solve (2.9) for the capillary surface with the boundary conditions $f^{\prime}(0)=-\cot \left(\theta_{1}\right)$ and $f^{\prime}(L)=\cot \left(\theta_{2}\right)$ without imposing a volume constraint, and the solution is shown in Figure 3. Next, we consider a motion of the left plate (that is plate 1 ) in the $y$ direction that is created by an external source, while the right end of the capillary surface is fixed at its initial height $f_{i}(L)$. Assume the final height of the left end of the capillary surface is $f_{f}(0)$. Then, a new capillary surface $f_{f}(x)$ is numerically obtained with the volume constraint by solving (2.9) with the new boundary conditions: $f(0)=f_{f}(0)$ and $f(L)=f_{i}(L)$. The modified simple shooting method, which is in [13], is used to solve the two-point boundary value problems. Parameter values used in the calculations are: surface tension of water $\gamma_{L G}=72 \mathrm{dyn} / \mathrm{cm}$; the gravitational acceleration $g=981 \mathrm{~cm} / \mathrm{s}^{2}$; the dynamic viscosity $\mu=0.8 \times 10^{-2} \mathrm{dyn} . \mathrm{s} / \mathrm{cm}^{-2}$; and the contact angles $\theta_{1}=50^{\circ}$ and $\theta_{2}=50^{\circ}$. 


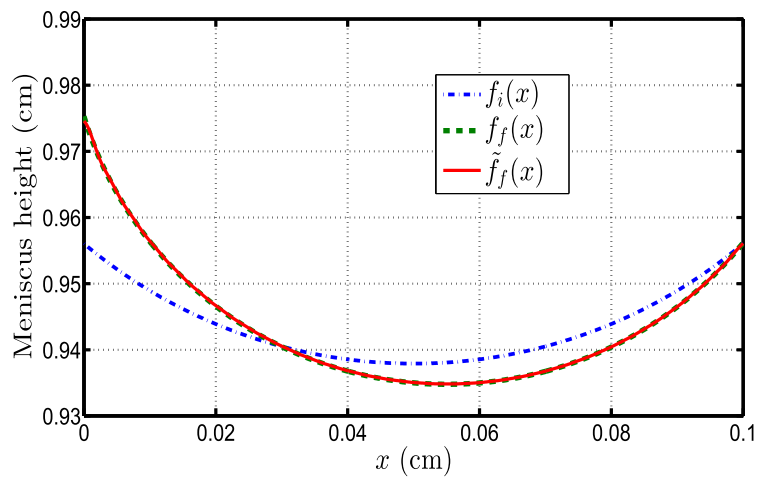

(a)

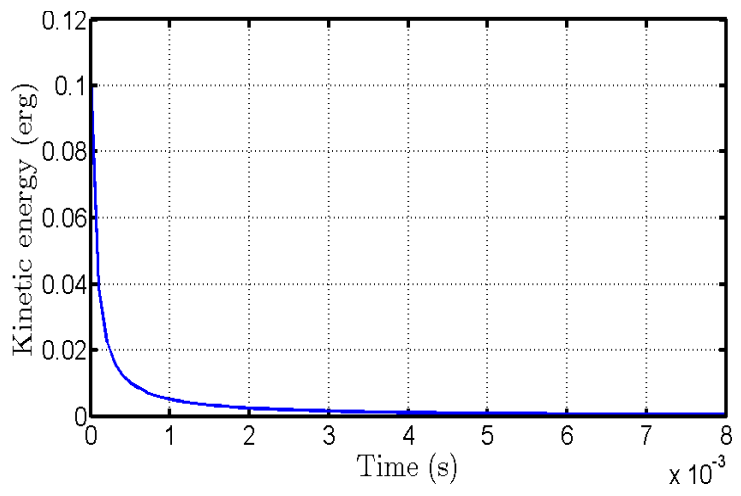

(b)

FiguRE 3. (a) Initial $\left(f_{i}(x)\right)$ and final $\left(f_{f}(x)\right)$ capillary surface profiles with the approximate solution $\left(\tilde{f}_{f}(x)\right)$ to $f_{f}(x)$. Observe that the capillary surface, $f_{i}(x)$, is symmetric due to the same contact angle values, and has maximum height $\left(\max \left\{f_{i}(x)\right\}=0.9560 \mathrm{~cm}\right)$ at the end points $x=0$ and $x=L$. Liquid volume between the capillary surface and the $x$-axis is $0.0944 \mathrm{~cm}^{3}$. $f_{f}(x)$ is obtained using the boundary conditions $y_{f}(0)=0.9753(\mathrm{~cm})$ and $f_{f}(L)=0.9560 \mathrm{~cm}$. The new contact angles $\theta_{f}(0)$ and $\theta_{f}(L)$ are $16.8^{\circ}$ and $38.9^{\circ}$, respectively. Liquid volume under the capillary surface is $0.0944 \mathrm{~cm}^{3}$, which has the same value as that of the curve $y_{i}(x)$. The approximate solution $\tilde{f}_{f}(x)$ for the meniscus profile is computed by solving the boundary value problem for the Navier-Stokes equations. $\left\|f_{f}(x)-\tilde{f}_{f}(x)\right\|_{2}=0.0025 \mathrm{~cm}$. Here, the number of Fourier coefficients, $N=60$, and the adjustable parameter $\epsilon=\eta$. (b) the initial kinetic energy variation with time.

\subsection{Numerical solutions for the initial velocity distribution}

Next, we consider the boundary value problem defined by the initial and the final capillary surfaces $f_{i}(x)$ and $f_{f}(x)$. The problem is to compute the initial velocity distribution that achieves the final capillary surface shape. Equation (3.14) is an implicit equation for $f(x)$. Hence, one may set up an algorithm to compute it. Having obtained the $f_{i}(x)$ and $f_{f}(x)$, we apply the Algorithm 4.1 to compute the solution to the Navier-Stokes equation. Our solution for the NS equation depends on the forms that we consider for velocity components $u(x, y, t)$ and $v(x, y, t)$. Note that these forms result in theoretically non-unique solutions.

Algorithm 4.1. Algorithm to compute a solution to the boundary value problem for the NS equation:

Step 1: Choose the number of Fourier coefficients $N$. Select an initial guess for the unknowns $u_{0}, \alpha_{1}(0), \widehat{\alpha}(0)$. There are exactly $N+1$ unknowns as $\alpha_{0}(0)$ must be 0 .

Step 2: Compute $f(x, t)$ according to:

$$
f(x, t)=f_{i}(x)+\int_{0}^{t}\left(v(x, y(x, s), s)-\frac{\partial f(x, s)}{\partial x} u(x, s)\right) \mathrm{d} s,
$$

where $v(x, y, t)=\frac{\alpha_{0}(t)}{2}+\sum_{k=1}^{N} \alpha_{k}(t) \cos \left(\frac{k \pi x}{L}\right)-\frac{\pi}{L} \cos \left(\frac{\pi x}{L}\right) u_{0}(t) y$.

Step 3: Due to the properties of $v$, the function $\int_{0}^{\infty} y(x, s) u_{0}(s) \mathrm{d} s$ is well-defined by Cauchy-Schwarz inequality. Compute the Fourier coefficients $\left\{a_{k}\right\}$ and $\left\{c_{k}\right\}$ using (4.1).

$$
\begin{aligned}
& f_{f}(x)-f_{i}(x)=\frac{a_{0}}{2}+\sum_{k=1}^{N} a_{k} \cos \left(\frac{k \pi x}{L}\right) \text { and } \\
& \frac{\pi}{L} \cos \left(\frac{\pi x}{L}\right) \int_{0}^{\infty} y(x, s) u_{0}(s) \mathrm{d} s+\int_{0}^{\infty} \frac{\partial f(x, t)}{\partial x} u(x, t) \mathrm{d} t=\left(\frac{c_{0}}{2}+\sum_{k=1}^{N} c_{k} \cos \left(\frac{k \pi x}{L}\right)\right) u_{0} .
\end{aligned}
$$


Step 4: Substitute (4.1) into (3.14), and obtain a system of $N+1$ equations of the form $a_{k}+u_{0} c_{k}=$ $\int_{0}^{\infty} \alpha_{k}(t) \mathrm{d} t 0 \leq k \leq N$. This resulted system is solved using Newton's method [19] for $u_{0}$ and $\alpha_{k}(0), 1 \leq k \leq N$. We denote this solution by $u_{0}^{(1)}$ and $\alpha_{k}^{(1)}(0), 1 \leq k \leq N$.

Step 5: Using the computed solution, we may compute $y^{(1)}(x, t)$ by going back to Step 2 and use it to compute $u_{0}^{(2)}$ and $\alpha_{k}^{(2)}(0), 1 \leq k \leq N$. The computations proceed until convergence is achieved for $f$.

Step 6: Stopping Criterion: Increase the value of $\mathrm{N}$ and recompute Steps 1 through 5. If the change in the dissipated energy due to viscosity and the kinetic energy (see Sect. 4.3) are within a prespecified tolerance then stop.

\subsection{Viscous energy dissipation}

Here, we numerically compute the viscous energy dissipation in the fluid flow that results from the deformation of the initial capillary surface to a final capillary surface. The presence of viscosity results in the dissipation of the energy during the fluid flow. Recall the fluid velocity of the flow $\mathbf{u}=(u(x, y, t), v(x, y, t))$ is

$$
\begin{aligned}
& u(x, y, t)=e^{-\eta t} u_{0} \sin \left(\frac{\pi x}{L}\right) \text { and } \\
& v(x, y, t)=\frac{\alpha_{0}(t)}{2}+\sum_{k=1}^{\infty} \alpha_{k}(t) \cos \left(\frac{k \pi x}{L}\right)-\frac{\pi}{L} e^{-\eta t} u_{0} \cos \left(\frac{\pi x}{L}\right) y .
\end{aligned}
$$

Then the viscous energy dissipation $D$ of the fluid flow with the viscosity $\mu[21]$ over the domain $[0, L]$ in the time interval $\left[0, t_{f}\right]$ is

$$
D=2 \mu \int_{0}^{L} \int_{0}^{t_{f}}\left[\left(\frac{\partial u}{\partial x}\right)^{2}+\left(\frac{\partial v}{\partial y}\right)^{2}-\frac{1}{3}(\nabla \cdot \mathbf{u})^{2}\right] \mathrm{d} t \mathrm{~d} x+\mu \int_{0}^{L} \int_{0}^{t_{f}}\left(\frac{\partial v}{\partial x}+\frac{\partial u}{\partial y}\right)^{2} \mathrm{~d} t \mathrm{~d} x .
$$

Then, we use (4.2), (4.3) and the continuity equation $\nabla \cdot \mathbf{u}=0$ to obtain

$$
2 \mu \int_{0}^{L} \int_{0}^{t_{f}}\left[\left(\frac{\partial u}{\partial x}\right)^{2}+\left(\frac{\partial v}{\partial y}\right)^{2}-\frac{1}{3}(\nabla \cdot \mathbf{u})^{2}\right] \mathrm{d} t \mathrm{~d} x=\frac{4 \mu \pi^{2} u_{0}^{2}}{L^{2}} \int_{0}^{L} \int_{0}^{t_{f}} e^{-2 \eta t} \cos ^{2}\left(\frac{\pi x}{L}\right) \mathrm{d} t \mathrm{~d} x
$$

and

$$
\mu \int_{0}^{L} \int_{0}^{t_{f}}\left(\frac{\partial v}{\partial x}+\frac{\partial u}{\partial y}\right)^{2} \mathrm{~d} t \mathrm{~d} x=\mu \int_{0}^{L} \int_{0}^{t_{f}}\left[\frac{\pi}{L} e^{-\eta t} u_{0} \sin \left(\frac{\pi x}{L}\right) y-\sum_{k=1}^{\infty} \frac{k \pi}{L} \alpha_{k}(t) \sin \left(\frac{k \pi x}{L}\right)\right]^{2} \mathrm{~d} t \mathrm{~d} x
$$

By considering the equations (4.4) and (4.5), the total energy dissipation is numerically computed with different numbers of Fourier coefficients, and the corresponding results are given in Table 1. Further, we observe the convergence of both the viscous dissipation and the initial kinetic energy values as the number of Fourier coefficients $N \rightarrow 60$. Initial velocity coefficients: $\left\{\alpha_{k}(0)\right\}$ values for $N=20,40,60$ are in Table 2 .

\subsection{Time-dependent Stokes flow}

In this section, we model the fluid flow that is in Section 3, using the time-dependent Stokes equation [8]. This is obtained by neglecting the inertial term $(\mathbf{u} \cdot \nabla \mathbf{u})$ from the Navier-Stokes equation, and hence; it may be expressed as

$$
\rho \frac{\partial \mathbf{u}}{\partial t}=\rho \mathbf{g}-\nabla p+\mu \nabla^{2} \mathbf{u}
$$


TABLE 1. Variations of viscous energy dissipation $D$ and the initial kinetic energy $K E^{i n i}$ with different number of Fourier coefficients $(N)$ values. Here, $t_{f}=1 \mathrm{~s}$.

\begin{tabular}{|c|c|c||c|c|c|}
\hline$N$ & $D \times 10^{-4}(\mathrm{erg})$ & $K E^{i n i}(\mathrm{erg})$ & $N$ & $D \times 10^{-4}(\mathrm{erg})$ & $K E^{\text {ini }}(\mathrm{erg})$ \\
\hline 10 & 2.21 & 0.008 & 50 & 29.00 & 0.099 \\
15 & 4.82 & 0.018 & 55 & 31.00 & 0.103 \\
20 & 7.48 & 0.029 & 57 & 31.00 & 0.103 \\
25 & 11.00 & 0.043 & 60 & 31.00 & 0.103 \\
\hline
\end{tabular}

TABLE 2. Initial velocity coefficients: $\left\{\alpha_{k}(0)\right\}$ values for $N=20,40,60$ for the Navier-Stokes fluid flow.

\begin{tabular}{|c|c|c|c||c|c|c||c|c|}
\hline & $N=20$ & $N=40$ & $N=60$ & & $N=40$ & $N=60$ & & $N=60$ \\
\hline$\alpha_{0}$ & 0.000 & 0.000 & 0.000 & $\alpha_{20}$ & 0.382 & 0.382 & $\alpha_{40}$ & 0.360 \\
$\alpha_{1}$ & 0.036 & 0.036 & 0.036 & $\alpha_{21}$ & 0.263 & 0.263 & $\alpha_{41}$ & 0.248 \\
$\alpha_{2}$ & 0.124 & 0.124 & 0.124 & $\alpha_{22}$ & 0.388 & 0.388 & $\alpha_{42}$ & 0.344 \\
$\alpha_{3}$ & 0.098 & 0.098 & 0.098 & $\alpha_{23}$ & 0.268 & 0.268 & $\alpha_{43}$ & 0.235 \\
$\alpha_{4}$ & 0.205 & 0.205 & 0.205 & $\alpha_{24}$ & 0.392 & 0.392 & $\alpha_{44}$ & 0.323 \\
$\alpha_{5}$ & 0.140 & 0.140 & 0.140 & $\alpha_{25}$ & 0.271 & 0.271 & $\alpha_{45}$ & 0.219 \\
$\alpha_{6}$ & 0.257 & 0.257 & 0.256 & $\alpha_{26}$ & 0.394 & 0.394 & $\alpha_{46}$ & 0.296 \\
$\alpha_{7}$ & 0.171 & 0.171 & 0.171 & $\alpha_{27}$ & 0.273 & 0.273 & $\alpha_{47}$ & 0.198 \\
$\alpha_{8}$ & 0.292 & 0.292 & 0.292 & $\alpha_{28}$ & 0.396 & 0.396 & $\alpha_{48}$ & 0.263 \\
$\alpha_{9}$ & 0.194 & 0.194 & 0.194 & $\alpha_{29}$ & 0.275 & 0.275 & $\alpha_{49}$ & 0.173 \\
$\alpha_{10}$ & 0.318 & 0.318 & 0.318 & $\alpha_{30}$ & 0.395 & 0.395 & $\alpha_{50}$ & 0.225 \\
$\alpha_{11}$ & 0.213 & 0.213 & 0.212 & $\alpha_{31}$ & 0.274 & 0.274 & $\alpha_{51}$ & 0.144 \\
$\alpha_{12}$ & 0.338 & 0.338 & 0.338 & $\alpha_{32}$ & 0.393 & 0.393 & $\alpha_{52}$ & 0.180 \\
$\alpha_{13}$ & 0.227 & 0.227 & 0.227 & $\alpha_{33}$ & 0.273 & 0.273 & $\alpha_{53}$ & 0.111 \\
$\alpha_{14}$ & 0.353 & 0.353 & 0.353 & $\alpha_{34}$ & 0.388 & 0.388 & $\alpha_{54}$ & 0.132 \\
$\alpha_{15}$ & 0.239 & 0.239 & 0.239 & $\alpha_{35}$ & 0.270 & 0.270 & $\alpha_{55}$ & 0.076 \\
$\alpha_{16}$ & 0.365 & 0.365 & 0.365 & $\alpha_{36}$ & 0.382 & 0.382 & $\alpha_{56}$ & 0.082 \\
$\alpha_{17}$ & 0.248 & 0.248 & 0.249 & $\alpha_{37}$ & 0.265 & 0.265 & $\alpha_{57}$ & 0.040 \\
$\alpha_{18}$ & 0.375 & 0.375 & 0.375 & $\alpha_{38}$ & 0.373 & 0.373 & $\alpha_{58}$ & 0.032 \\
$\alpha_{19}$ & 0.257 & 0.257 & 0.256 & $\alpha_{39}$ & 0.258 & 0.258 & $\alpha_{59}$ & 0.006 \\
\hline
\end{tabular}

TABLE 3. Variations of $K E^{i n i}$ and $D$ with different number of Fourier coefficients $(N)$ values for the time-dependent Stokes flow.

\begin{tabular}{|c|c|c||c|c|c|}
\hline$N$ & $D \times 10^{-4}(\mathrm{erg})$ & $K E^{i n i}(\mathrm{erg})$ & $N$ & $D \times 10^{-4}(\mathrm{erg})$ & $K E^{\text {ini }}(\mathrm{erg})$ \\
\hline 10 & 13.00 & 0.014 & 50 & 42.00 & 0.115 \\
20 & 19.00 & 0.038 & 55 & 42.00 & 0.115 \\
30 & 26.00 & 0.067 & 60 & 42.00 & 0.115 \\
40 & 35.00 & 0.096 & & & \\
\hline
\end{tabular}

We use the same velocity components

$$
u(x, y, t)=e^{-\eta t} u_{0} \sin \left(\frac{\pi x}{L}\right) \quad \text { and } \quad v(x, y, t)=\frac{\alpha_{0}(t)}{2}+\sum_{k=1}^{\infty} \alpha_{k}(t) \cos \left(\frac{k \pi x}{L}\right)-\frac{\pi}{L} e^{-\eta t} u_{0} \cos \left(\frac{\pi x}{L}\right) y .
$$


TABLE 4. Initial velocity coefficients: $\left\{\alpha_{k}(0)\right\}$ values for $N=20,40,60$ for the time-dependent Stokes flow.

\begin{tabular}{|c|c|c|c||c|c|c||c|c|}
\hline & $N=20$ & $N=40$ & $N=60$ & & $N=40$ & $N=60$ & & $N=60$ \\
\hline$\alpha_{0}$ & 0.000 & 0.000 & 0.000 & $\alpha_{20}$ & 0.292 & 0.292 & $\alpha_{40}$ & 0.270 \\
$\alpha_{1}$ & 0.126 & 0.126 & 0.126 & $\alpha_{21}$ & 0.391 & 0.391 & $\alpha_{41}$ & 0.350 \\
$\alpha_{2}$ & 0.131 & 0.131 & 0.131 & $\alpha_{22}$ & 0.297 & 0.297 & $\alpha_{42}$ & 0.256 \\
$\alpha_{3}$ & 0.207 & 0.207 & 0.207 & $\alpha_{23}$ & 0.396 & 0.396 & $\alpha_{43}$ & 0.329 \\
$\alpha_{4}$ & 0.169 & 0.169 & 0.169 & $\alpha_{24}$ & 0.300 & 0.300 & $\alpha_{44}$ & 0.237 \\
$\alpha_{5}$ & 0.258 & 0.258 & 0.258 & $\alpha_{25}$ & 0.398 & 0.398 & $\alpha_{45}$ & 0.302 \\
$\alpha_{6}$ & 0.200 & 0.200 & 0.200 & $\alpha_{26}$ & 0.302 & 0.302 & $\alpha_{46}$ & 0.214 \\
$\alpha_{7}$ & 0.294 & 0.294 & 0.294 & $\alpha_{27}$ & 0.399 & 0.399 & $\alpha_{47}$ & 0.269 \\
$\alpha_{8}$ & 0.223 & 0.223 & 0.223 & $\alpha_{28}$ & 0.303 & 0.303 & $\alpha_{48}$ & 0.186 \\
$\alpha_{9}$ & 0.320 & 0.320 & 0.320 & $\alpha_{29}$ & 0.399 & 0.399 & $\alpha_{49}$ & 0.230 \\
$\alpha_{10}$ & 0.242 & 0.242 & 0.242 & $\alpha_{30}$ & 0.302 & 0.302 & $\alpha_{50}$ & 0.154 \\
$\alpha_{11}$ & 0.340 & 0.340 & 0.340 & $\alpha_{31}$ & 0.397 & 0.397 & $\alpha_{51}$ & 0.185 \\
$\alpha_{12}$ & 0.257 & 0.257 & 0.257 & $\alpha_{32}$ & 0.300 & 0.300 & $\alpha_{52}$ & 0.118 \\
$\alpha_{13}$ & 0.356 & 0.356 & 0.356 & $\alpha_{33}$ & 0.393 & 0.393 & $\alpha_{53}$ & 0.136 \\
$\alpha_{14}$ & 0.268 & 0.268 & 0.268 & $\alpha_{34}$ & 0.296 & 0.296 & $\alpha_{54}$ & 0.081 \\
$\alpha_{15}$ & 0.368 & 0.368 & 0.368 & $\alpha_{35}$ & 0.387 & 0.387 & $\alpha_{55}$ & 0.084 \\
$\alpha_{16}$ & 0.278 & 0.278 & 0.278 & $\alpha_{36}$ & 0.290 & 0.290 & $\alpha_{56}$ & 0.043 \\
$\alpha_{17}$ & 0.378 & 0.378 & 0.378 & $\alpha_{37}$ & 0.378 & 0.378 & $\alpha_{57}$ & 0.036 \\
$\alpha_{18}$ & 0.286 & 0.286 & 0.286 & $\alpha_{38}$ & 0.281 & 0.281 & $\alpha_{58}$ & 0.007 \\
$\alpha_{19}$ & 0.385 & 0.385 & 0.385 & $\alpha_{39}$ & 0.366 & 0.366 & $\alpha_{59}$ & 0.002 \\
\hline
\end{tabular}

to describe the fluid flow $\mathbf{u}=(u(x, y, t), v(x, y, t))$. Then, we numerically compute the viscous energy dissipation and the initial velocity coefficient, and the corresponding results are illustrated in Tables 3 and 4 , respectively. Based on the results, we observe that the Stokes equation overestimates the energy dissipation due to viscosity compared to the Navier-Stokes equation.

\section{Conclusions}

In this paper, we investigated the dynamic motion of a capillary surface that forms between two vertical plates. The initial and the final capillary surfaces were modeled using a calculus of variations approach with a volume constraint. We theoretically studied the full Navier-Stokes equation for the boundary value problem for fluid motion that deforms the capillary surface using a Fourier series method. We showed that there are non-unique solutions to the problem. We computed a central solution whose perturbation yields other solutions.

\section{Appendix A. Preliminary Results}

\section{A.1 Proof of Lemma 3.1}

Proof. Let $r(t)^{2}=\left\|\widehat{\alpha}_{0}(t)\right\|_{2}^{2}$. Then the derivative of $r$ with respect to $t$, results in

$$
r r^{\prime}=\alpha_{0} \alpha_{0}^{\prime}+\alpha_{1} \alpha_{1}^{\prime}=\left(-\epsilon \alpha_{0}^{2}-\eta \alpha_{1}^{2}\right)+5 \varphi \alpha_{0} \alpha_{1} \leq\left(-\epsilon \alpha_{0}^{2}-\eta \alpha_{1}^{2}\right)+\frac{5}{2}|\varphi|\left(\alpha_{0}^{2}+\alpha_{1}^{2}\right)
$$


Choose $\epsilon=\eta$. Hence, we get

$$
\begin{aligned}
r r^{\prime} & \leq-\eta\left(\alpha_{0}^{2}+\alpha_{1}^{2}\right)+\frac{5}{2}|\varphi|\left(\alpha_{0}^{2}+\alpha_{1}^{2}\right), \\
r^{\prime} & \leq\left(-\eta+\frac{5|\varphi|}{2}\right) r .
\end{aligned}
$$

By letting $\zeta^{\prime}(t)=\left(-\eta+\frac{5|\varphi|}{2}\right) \zeta(t)$ with $\zeta(0)=r(0)$ and using the comparison lemma [15], one may obtain

$$
r(t)=\left\|\widehat{\alpha}_{0}(t)\right\|_{2} \leq \widetilde{k}_{1} e^{-\widetilde{k}_{2} t}\left\|\widehat{\alpha}_{0}(0)\right\|_{2}
$$

where $\widetilde{k}_{1}=e^{\frac{5 \pi\left|u_{0}\right|}{4 L \eta}}$ and $\widetilde{k}_{2}=\eta$.

\section{A.2 Proof of Lemma 3.2}

Proof. Let $r^{2}(t)=\left\|\widehat{\alpha}_{N}\right\|_{2}^{2}=\alpha_{2}^{2}(t)+\alpha_{3}^{2}(t)+\cdots+\alpha_{N}^{2}(t)$. Then, the derivative of $r$ with respect to $t$ variable results,

$$
\begin{aligned}
r r^{\prime}= & \alpha_{2} \alpha_{2}^{\prime}+\alpha_{3} \alpha_{3}^{\prime}+\cdots+\alpha_{N} \alpha_{N}^{\prime} \\
= & \alpha_{2}\left(-4 \eta \alpha_{2}+4 \varphi(t) \alpha_{3}\right)+\alpha_{3}\left(-\varphi(t) \alpha_{2}-9 \eta \alpha_{3}+5 \varphi(t) \alpha_{4}\right)+\ldots \\
& +\alpha_{N}\left[-(N-2) \varphi(t) \alpha_{N-1}-N^{2} \eta \alpha_{N}\right] \\
\leq & \left(-4 \eta \alpha_{2}^{2}-9 \eta \alpha_{3}^{2}-\cdots-N^{2} \eta \alpha_{N}^{2}\right)+3 \varphi(t)\left[\alpha_{2} \alpha_{3}+\alpha_{3} \alpha_{4}+\cdots+\alpha_{N-1} \alpha_{N}\right] \\
\leq & -4 \eta\left(\alpha_{2}^{2}+\cdots+\alpha_{N}^{2}\right)+3|\varphi(t)|\left[\frac{\alpha_{2}^{2}+\alpha_{3}^{2}}{2}+\frac{\alpha_{3}^{2}+\alpha_{4}^{2}}{2}+\cdots+\frac{\alpha_{N-1}^{2}+\alpha_{N}^{2}}{2}\right], \\
\leq & -4 \eta\left(\alpha_{2}^{2}+\cdots+\alpha_{N}^{2}\right)+3|\varphi(t)|\left[\alpha_{2}^{2}+\alpha_{3}^{2}+\cdots+\alpha_{N}^{2}\right]=(-4 \eta+3|\varphi(t)|) r^{2} .
\end{aligned}
$$

Hence, we obtain the inequality: $r^{\prime} \leq(-4 \eta+3|\varphi(t)|) r$, with $\eta=\frac{\mu \pi^{2}}{\rho L^{2}}, \varphi(t)=\frac{\pi u_{0}(t)}{2 L}$, and $u_{0}(t)=e^{-\eta t} u_{0}$. Let $\zeta^{\prime}=(-4 \eta+3|\varphi(t)|) \zeta$ with $\zeta(0)=r(0)$. Then,

$$
\zeta(t)=e^{\int_{0}^{t}(-4 \eta+3|\varphi(s)|) \mathrm{d} s} \zeta(0)=e^{-4 \eta t+\frac{3 \pi}{2 L \eta}\left|u_{0}\right|\left(1-e^{-\eta t}\right)} \zeta(0) \leq k_{1} e^{-4 \eta t} \zeta(0),
$$

where $k_{1}=e^{\frac{3 \pi}{2 L \eta}\left|u_{0}\right|}$. Thus, from the comparison lemma [15], we obtain

$$
\left\|\widehat{\alpha}_{N}(t)\right\|_{2} \leq \zeta(t) \leq k_{1} e^{-k_{2} t}\left\|\widehat{\alpha}_{N}(0)\right\|_{2}
$$

where $k_{2}=4 \eta$ and $k_{1}$ and $k_{2}$ do not depend on $N$.

Hence, if $\widehat{\alpha}(0)=\left(\alpha_{2}(0), \alpha_{3}(0), \ldots\right) \in l^{2}$,

$$
\begin{array}{r}
\left\|\widehat{\alpha}_{N}(t)\right\|_{2} \leq k_{1} e^{-k_{2} t}\left\|\widehat{\alpha}_{N}(0)\right\|_{2} \leq k_{1} e^{-k_{2} t}\|\widehat{\alpha}(0)\|_{2}, \\
\left\|\widehat{\alpha}_{N}(t)\right\|_{2} \leq k_{1} e^{-k_{2} t}\|\widehat{\alpha}(0)\|_{2} \quad \forall t>0,
\end{array}
$$

and this conclude the proof of Lemma 3.2. 


\section{A.3 Proof of Lemma 3.3}

Proof. Let the solution of the system (3.19) be

$$
\left[\begin{array}{c}
\alpha_{0}(t) \\
\alpha_{1}(t)
\end{array}\right]=\Phi(t, 0)\left[\begin{array}{c}
\alpha_{0}(0) \\
\alpha_{1}(0)
\end{array}\right]+\int_{0}^{t} \Phi(t, s)\left[\begin{array}{c}
0 \\
\alpha_{2}(s)
\end{array}\right] \mathrm{d} s
$$

where $\Phi(t, s)$ is the transition matrix of the system (3.18). By using Lemmas 3.1 and 3.2, we get

$$
\begin{aligned}
\left\|\widehat{\alpha}_{0}(t)\right\|_{2} & \leq \widetilde{k}_{1} e^{-\widetilde{k}_{2} t}\left\|\widehat{\alpha}_{0}(0)\right\|_{2}+\int_{0}^{t} \widetilde{k}_{1} e^{-\widetilde{k}_{2}(t-s)} k_{1} e^{-k_{2} s}\left\|\widehat{\alpha}_{N}(0)\right\| \mathrm{d} s \\
& =\widetilde{k}_{1} e^{-\widetilde{k}_{2} t}\left\|\widehat{\alpha}_{0}(0)\right\|_{2}+\frac{\widetilde{k}_{1} k_{1}}{k_{2}-\widetilde{k}_{2}}\left(e^{-\widetilde{k}_{2} t}-e^{-k_{2} t}\right)\left\|\widehat{\alpha}_{N}(0)\right\| .
\end{aligned}
$$

Moreover, using Lemmas 3.1 and 3.2, we get

$$
\|\widehat{\alpha}(t)\|_{2}^{2}=\alpha_{0}^{2}(t)+\alpha_{1}^{2}(t)+\alpha_{2}^{2}(t)+\cdots+\alpha_{N}^{2}(t) \leq \widetilde{k}_{1}^{2} e^{-2 \widetilde{k}_{2} t}\left\|\widehat{\alpha}_{0}(0)\right\|_{2}^{2}+k_{1}^{2} e^{-2 k_{2}}\left\|\widehat{\alpha}_{N}(0)\right\|_{2}^{2} .
$$

Letting $K_{1}=\max \left\{k_{1}, \widetilde{k}_{1}\right\}$ and $K_{2}=\min \left\{k_{2}, \widetilde{k}_{2}\right\}$, one gets

$$
\|\widehat{\alpha}(t)\|_{2}^{2} \leq K_{1}^{2} e^{-2 K_{2}}\left(\left\|\widehat{\alpha}_{0}(0)\right\|_{2}^{2}+\left\|\widehat{\alpha}_{N}(0)\right\|_{2}^{2}\right)=K_{1}^{2} e^{-2 K_{2}}\|\widehat{\alpha}(0)\|_{2}^{2},
$$

and hence, $\|\widehat{\alpha}(t)\|_{2} \leq K_{1} e^{-K_{2}}\|\widehat{\alpha}(0)\|_{2}$.

\section{A.4 Proof of Lemma 3.7}

Proof. Let $r^{2}(t)=\left\|\widehat{\lambda}_{N}\right\|_{2}^{2}=\lambda_{2}^{2}(t)+\lambda_{3}^{2}(t)+\cdots+\lambda_{N}^{2}(t)$. Then, the derivative with respect to $t$ variable results,

$$
\begin{aligned}
r r^{\prime}= & \lambda_{2} \lambda_{2}^{\prime}+\lambda_{3} \lambda_{3}^{\prime}+\cdots+\lambda_{N} \lambda_{N}^{\prime}=2^{4} \lambda_{2} \alpha_{2}^{\prime}+3^{4} \lambda_{3} \alpha_{3}^{\prime}+\cdots+N^{4} \lambda_{N} \alpha_{N}^{\prime}, \\
= & 2^{4} \lambda_{2}\left(-4 \eta \alpha_{2}+4 \varphi(t) \alpha_{3}\right)+3^{4} \lambda_{3}\left(-\varphi(t) \alpha_{2}-9 \eta \alpha_{3}+5 \varphi(t) \alpha_{4}\right)+\ldots \\
& +N^{4} \beta_{N}\left[-(N-2) \varphi(t) \alpha_{N-1}-N^{2} \eta \alpha_{N}\right], \\
\leq & -4 \eta\left(\lambda_{2}^{2}+\cdots+\lambda_{N}^{2}\right)+\left(\frac{4 \cdot 2^{4}}{3^{4}}-\frac{3^{4}}{2^{4}}\right) \varphi \lambda_{2} \lambda_{3}+\left(\frac{5 \cdot 3^{4}}{4^{4}}-\frac{2 \cdot 4^{4}}{3^{4}}\right) \varphi \lambda_{3} \lambda_{4}+\ldots \\
& +\left(\frac{(N-1)^{4}(N+1)}{N^{4}}-\frac{N^{4}(N-2)}{(N-1)^{4}}\right) \varphi \lambda_{N-1} \lambda_{N} \\
\leq & -4 \eta\left(\lambda_{2}^{2}+\cdots+\lambda_{N}^{2}\right)-\frac{5537}{1296}|\varphi(t)|\left[\frac{\lambda_{2}^{2}+\lambda_{3}^{2}}{2}+\frac{\lambda_{3}^{2}+\lambda_{4}^{2}}{2}+\cdots+\frac{\lambda_{N-1}^{2}+\lambda_{N}^{2}}{2}\right], \\
\leq & -4 \eta\left(\lambda_{2}^{2}+\cdots+\lambda_{N}^{2}\right)-\frac{5537}{1296}|\varphi(t)|\left(\lambda_{2}^{2}+\lambda_{3}^{2}+\cdots+\lambda_{N}^{2}\right) \\
\leq & \left(-4 \eta+\frac{5537}{1296}|\varphi(t)|\right) r^{2} .
\end{aligned}
$$

At this point, mimic the proof of Lemma 3.2. Further, $\widehat{\lambda}(0)=\left(\lambda_{2}(0), \lambda_{3}(0), \ldots\right) \in l^{2}$, then $\exists p_{1}$ and $p_{2}$ so that

$$
\begin{aligned}
&\left\|\widehat{\lambda}_{N}(t)\right\|_{2} \leq p_{1} e^{-p_{2} t}\left\|\widehat{\lambda}_{N}(0)\right\|_{2} \leq p_{1} e^{-p_{2} t}\|\widehat{\lambda}(0)\|_{2}, \text { and } \\
&\|\widehat{\lambda}(t)\|_{2} \leq p_{1} e^{-p_{2} t}\|\widehat{\lambda}(0)\|_{2} \quad \forall t>0 .
\end{aligned}
$$


Lemma A.1. Consider the Fourier series:

$$
A(x, t)=\frac{\alpha_{0}(t)}{2}+\sum_{k=1}^{\infty} \alpha_{k}(t) \cos \left(\frac{k \pi x}{L}\right) .
$$

Then, its mixed partial derivatives are in $L^{2}((0, \infty) \times[0, L])$.

Proof. $A(x, t) \in L^{2}([0, L] \times(0, \infty))$

$$
\int_{0}^{\infty} \int_{0}^{L} A^{2}(x, t) \mathrm{d} x \mathrm{~d} t=\int_{0}^{\infty} \int_{0}^{L}\left(\frac{\alpha_{0}(t)}{2}+\sum_{k=1}^{\infty} \alpha_{k}(t) \cos \left(\frac{k \pi x}{L}\right)\right)^{2} \mathrm{~d} x \mathrm{~d} t .
$$

Using Parserval-Plancherel theorem and (3.21), we obtain

$$
\int_{0}^{\infty} \int_{0}^{L} A^{2}(x, t) \mathrm{d} x \mathrm{~d} t=\int_{0}^{\infty}\left\|\alpha_{k}(t)\right\|_{2}^{2} \mathrm{~d} t \leq \frac{K_{1}^{2}}{2 K_{2}}\|\widehat{\alpha}(0)\|_{2}^{2}
$$

$A_{x}(x, t) \in L^{2}([0, L] \times(0, \infty))$

$$
\int_{0}^{\infty} \int_{0}^{L} A_{x}^{2}(x, t) \mathrm{d} x \mathrm{~d} t=\int_{0}^{\infty} \int_{0}^{L}\left(\frac{\alpha_{0}(t)}{2}+\sum_{k=1}^{\infty} \frac{k \pi}{L} \alpha_{k}(t) \sin \left(\frac{k \pi x}{L}\right)\right)^{2} \mathrm{~d} x \mathrm{~d} t \leq \frac{\pi^{2} c_{1}^{2}}{2 c_{2} L}\|\widehat{\theta}(0)\|_{2}^{2},
$$

where the last inequality results from Lemma 3.4. $A_{x x}(x, t) \in L^{2}([0, L] \times(0, \infty))$

$$
\int_{0}^{\infty} \int_{0}^{L} A_{x x}^{2}(x, t) \mathrm{d} x \mathrm{~d} t=\int_{0}^{\infty} \int_{0}^{L}\left(\sum_{k=1}^{\infty} \frac{k^{2} \pi^{2}}{L^{2}} \alpha_{k}(t) \cos \left(\frac{k \pi x}{L}\right)\right)^{2} \mathrm{~d} x \mathrm{~d} t \leq \frac{\pi^{4} m_{1}^{2}}{2 m_{2} L^{4}}\|\widehat{\gamma}(0)\|_{2}^{2},
$$

where the last inequality results from Lemma 3.5.

$$
A_{t}(x, t) \in L^{2}([0, L] \times(0, \infty))
$$

$$
\begin{aligned}
\int_{0}^{\infty} \int_{0}^{L} A_{t}^{2}(x, t) \mathrm{d} x \mathrm{~d} t & =\int_{0}^{\infty} \int_{0}^{L}\left(\frac{\alpha_{0}^{\prime}(t)}{2}+\sum_{k=1}^{\infty} \alpha_{k}^{\prime}(t) \cos \left(\frac{k \pi x}{L}\right)\right)^{2} \mathrm{~d} x \mathrm{~d} t \\
& =\int_{0}^{\infty}\left\|\alpha_{k}^{\prime}(t)\right\|_{2}^{2} \mathrm{~d} t=\int_{0}^{\infty}\left|\alpha_{0}^{\prime}(t)\right|^{2} \mathrm{~d} t+\int_{0}^{\infty} \sum_{k=1}^{\infty}\left|\alpha_{k}^{\prime}(t)\right|^{2} \mathrm{~d} t
\end{aligned}
$$

where the $\alpha_{k}^{\prime}(t)$ terms are in the system (3.15), and in general, $\alpha_{k}^{\prime}(t)$ may be written as

$$
\alpha_{k}^{\prime}(t)=-(k-2) \varphi(t) \alpha_{k-1}(t)-k^{2} \eta \alpha_{k}(t)+(k+2) \varphi(t) \alpha_{k+1}(t) \quad \forall k \geq 1,
$$

that can be recast into

$$
\alpha_{k}^{\prime}(t)=-(k-1) \varphi(t) \alpha_{k-1}(t)-k^{2} \eta \alpha_{k}(t)+(k+1) \varphi(t) \alpha_{k+1}(t)+\varphi(t)\left(\alpha_{k-1}(t)+\alpha_{k+1}(t)\right)
$$

Hence,

$$
\begin{aligned}
\left|\alpha_{k}^{\prime}(t)\right|^{2} & =\left[-(k-1) \varphi(t) \alpha_{k-1}(t)-k^{2} \eta \alpha_{k}(t)+(k+1) \varphi(t) \alpha_{k+1}(t)+\varphi(t)\left(\alpha_{k-1}(t)+\alpha_{k+1}(t)\right)\right]^{2} \\
& \leq 2\left[\left(-(k-1) \varphi \alpha_{k-1}(t)-k^{2} \eta \alpha_{k}(t)+(k+1) \varphi \alpha_{k+1}(t)\right)^{2}+\varphi\left(\alpha_{k-1}(t)+\alpha_{k+1}(t)\right)^{2}\right] \\
& \leq 6\left[(k-1)^{2} \varphi^{2} \alpha_{k-1}^{2}+k^{4} \eta^{2} \alpha_{k}^{2}+(k+1)^{2} \varphi^{2} \alpha_{k+1}^{2}\right]+4 \varphi^{2}\left(\alpha_{k-1}^{2}+\alpha_{k+1}^{2}\right) .
\end{aligned}
$$


Now, let us obtain an inequality for the term

$$
\begin{aligned}
\sum_{k=1}^{\infty}\left|\alpha_{k}^{\prime}(t)\right|^{2} \leq & \sum_{k=1}^{\infty} 6\left[(k-1)^{2} \varphi^{2} \alpha_{k-1}^{2}+k^{4} \eta^{2} \alpha_{k}^{2}+(k+1)^{2} \varphi^{2} \alpha_{k+1}^{2}\right] \\
& +4 \varphi^{2}\left(\alpha_{k-1}^{2}+\alpha_{k+1}^{2}\right) \\
\leq & 12 \varphi^{2}(t) \sum_{k=1}^{\infty}\left(k \alpha_{k}\right)^{2}+6 \eta^{2} \sum_{k=1}^{\infty}\left(k^{2} \alpha_{k}\right)^{2}+8 \varphi^{2}(t) \sum_{k=1}^{\infty}\left(\alpha_{k}\right)^{2} \\
\leq & \left(20 \varphi^{2}(t)+6 \eta^{2}\right) \sum_{k=1}^{\infty}\left(k^{2} \alpha_{k}\right)^{2} \leq\left(20 \varphi^{2}(t)+6 \eta^{2}\right) m_{1}^{2} e^{-2 m_{2} t}\|\widehat{\gamma}(0)\|_{2}^{2}
\end{aligned}
$$

where the last estimates are obtained using Lemma 3.5. Now, using the monotonicity of integration, we have

$$
\begin{aligned}
\int_{0}^{\infty} \sum_{k=1}^{\infty}\left|\alpha_{k}^{\prime}(t)\right|^{2} \mathrm{~d} t & \leq \int_{0}^{\infty}\left(20 \varphi^{2}(t)+6 \eta^{2}\right) m_{1}^{2} e^{-2 m_{2} t}\|\widehat{\gamma}(0)\|_{2}^{2} \\
& \leq\left(\frac{5 \pi^{2} u_{0}^{2} m_{1}^{2}}{L^{2}\left(\eta+m_{2}\right)}+\frac{6 \eta^{2} m_{1}^{2}}{m_{2}}\right)\|\widehat{\gamma}(0)\|_{2}^{2}
\end{aligned}
$$

Consider the term

$$
\begin{aligned}
\left|\alpha_{0}^{\prime}(t)\right|^{2} & =\left(-\epsilon \alpha_{0}(t)+4 \varphi \alpha_{1}(t)\right)^{2} \leq k^{*}\left(\alpha_{0}^{2}+\alpha_{1}^{2}\right) \leq k^{*}{\widetilde{k_{1}}}^{2} e^{-2 \widetilde{k_{2}} t}\left\|\widehat{\alpha}_{0}(0)\right\|_{2}^{2} \\
& =\frac{k^{*}{\widetilde{k_{1}}}^{2}}{2 \widetilde{k_{2}}}\left\|\widehat{\alpha}_{0}(0)\right\|_{2}^{2},
\end{aligned}
$$

and $k^{*}=\max \left\{\epsilon^{2}-4 \epsilon \varphi(t), 16 \varphi(t)-4 \epsilon \varphi(t)\right\}$. Therefore,

$$
\int_{0}^{\infty} \int_{0}^{L} A_{t}^{2}(x, t) \mathrm{d} x \mathrm{~d} t \leq \frac{k^{*}{\widetilde{k_{1}}}^{2}}{2 \widetilde{k_{2}}}\left\|\widehat{\alpha}_{0}(0)\right\|_{2}^{2}+\left(\frac{5 \pi^{2} u_{0}^{2} m_{1}^{2}}{L^{2}\left(\eta+m_{2}\right)}+\frac{6 \eta^{2} m_{1}^{2}}{m_{2}}\right)\|\widehat{\gamma}(0)\|_{2}^{2} .
$$

$A_{t x}(x, t) \in L^{2}([0, L] \times(0, \infty))$

$$
\begin{aligned}
\int_{0}^{\infty} \int_{0}^{L} A_{t x}^{2}(x, t) \mathrm{d} x \mathrm{~d} t & =\int_{0}^{\infty} \int_{0}^{L}\left(\sum_{k=1}^{\infty} \frac{k \pi}{L} \alpha_{k}^{\prime}(t) \sin \left(\frac{k \pi x}{L}\right)\right)^{2} \mathrm{~d} x \mathrm{~d} t \\
& \leq \int_{0}^{\infty} \frac{\pi^{2}}{L^{2}}\left\|k^{2} \alpha_{k}^{\prime}(t)\right\|_{2}^{2}
\end{aligned}
$$

where, we use the fact that $\left\|k \alpha_{k}^{\prime}(t)\right\|_{2}^{2}<\left\|k^{2} \alpha_{k}^{\prime}(t)\right\|_{2}^{2}$. Now, consider

$$
\begin{aligned}
\left(k^{2} \alpha_{k}^{\prime}\right)^{2} & =k^{4}\left((k-1) \varphi \alpha_{k-1}+\eta k^{2} \alpha_{k}+(k+1) \varphi \alpha_{k+1}+\varphi\left(\alpha_{k-1}+\alpha_{k+1}\right)\right)^{2} \\
& \leq 2\left[3 k^{4}\left((k-1)^{2} \varphi^{2} \alpha_{k-1}^{2}+\eta^{2} k^{4} \alpha_{k}^{2}+(k+1)^{2} \varphi^{2} \alpha_{k+1}^{2}\right)+2 \varphi^{2} k^{4}\left(\alpha_{k-1}^{2}+\alpha_{k+1}^{2}\right)\right] \\
& \leq 10 \varphi^{2} k^{4}(k-1)^{2} \alpha_{k-1}^{2}+10 \varphi^{2} k^{4}(k+1)^{2} \alpha_{k+1}^{2}+6 \eta^{2}\left(k^{4} \alpha_{k}\right)^{2}
\end{aligned}
$$


Note that for $k \geq 2$,

$$
\left(k^{2} \alpha_{k}^{\prime}\right)^{2} \leq 180 \varphi^{2}(k-1)^{6} \alpha_{k-1}^{2}+10 \varphi^{2}(k+1)^{6} \alpha_{k+1}^{2}+6 \eta^{2}\left(k^{4} \alpha_{k}\right)^{2} .
$$

Therefore, by re-indexing the first two terms, we have

$$
\sum_{k=1}^{\infty}\left(k^{2} \alpha_{k}^{\prime}\right)^{2} \leq 360 \varphi^{2} \sum_{k=1}^{\infty}\left(k^{3} \alpha_{k}\right)^{2}+\sum_{k=1}^{\infty} 6 \eta^{2}\left(k^{4} \alpha_{k}\right)^{2} .
$$

The convergence of the first and second series can be proved using Lemmas 3.6 and 3.7, respectively.

$$
\begin{aligned}
\int_{0}^{\infty} \sum_{k=1}^{\infty}\left(k^{2} \alpha_{k}^{\prime}\right)^{2} \mathrm{~d} t & \leq \int_{0}^{\infty}\left(360 \varphi^{2}(t) a_{1}^{2} e^{-2 a_{2} t}\|\widehat{\beta}(0)\|_{2}^{2}+6 \eta^{2} \lambda_{1}^{2} e^{-2 \lambda_{2} t}\|\widehat{\lambda}(0)\|_{2}^{2}\right) \mathrm{d} t \\
& =\frac{360 \pi^{2}\left|\bar{u}_{0}\right|^{2} a_{1}^{2}}{4 L^{2} 2\left(\eta+a_{2}\right)}\|\widehat{\beta}(0)\|_{2}^{2}+\frac{6 \eta^{2} \lambda_{1}^{2}}{2 \lambda_{2}}\|\widehat{\lambda}(0)\|_{2}^{2}
\end{aligned}
$$

Therefore,

$$
\int_{0}^{\infty} \int_{0}^{L} A_{t x}^{2}(x, t) \mathrm{d} x \mathrm{~d} t \leq \frac{\pi^{2}}{L^{2}}\left[\frac{360 \pi^{2}\left|\bar{u}_{0}\right|^{2} a_{1}^{2}}{4 L^{2} 2\left(\eta+a_{2}\right)}\|\widehat{\beta}(0)\|_{2}^{2}+\frac{6 \eta^{2} \lambda_{1}^{2}}{2 \lambda_{2}}\|\widehat{\lambda}(0)\|_{2}^{2}\right]
$$

$A_{t t} \in L^{2}([0, L] \times(0, \infty))$

$$
\begin{aligned}
\int_{0}^{\infty} \int_{0}^{L} A_{t t}^{2}(x, t) \mathrm{d} x \mathrm{~d} t & =\int_{0}^{\infty} \int_{0}^{L}\left(\frac{\alpha_{0}^{\prime \prime}(t)}{2}+\sum_{k=1}^{\infty} \alpha_{k}^{\prime \prime}(t) \cos \left(\frac{k \pi x}{L}\right)\right)^{2} \mathrm{~d} x \mathrm{~d} t \\
& =\int_{0}^{\infty}\left\|\alpha_{k}^{\prime \prime}(t)\right\|_{2}^{2} \mathrm{~d} t=\int_{0}^{\infty}\left|\alpha_{0}^{\prime \prime}(t)\right|^{2} \mathrm{~d} t+\int_{0}^{\infty} \sum_{k=1}^{\infty}\left|\alpha_{k}^{\prime \prime}(t)\right|^{2} \mathrm{~d} t .
\end{aligned}
$$

Consider

$$
\begin{aligned}
\sum_{k=1}^{\infty}\left|\alpha_{k}^{\prime \prime}(t)\right|^{2}= & \sum_{k=1}^{\infty}\left(\left[-(k-1) \varphi \alpha_{k-1}^{\prime}-k^{2} \eta \alpha_{k}^{\prime}+(k+1) \varphi \alpha_{k+1}^{\prime}\right]+\left[\varphi\left(\alpha_{k-1}^{\prime}+\alpha_{k+1}^{\prime}\right)\right]\right. \\
& \left.-\left[\varphi^{\prime}\left((k-1) \alpha_{k-1}+(k+1) \alpha_{k+1}\right)\right]+\left[\varphi^{\prime}\left(\alpha_{k-1}+\alpha_{k+1}\right)\right]\right)^{2} \\
\leq & 4 \sum_{k=1}^{\infty}\left(3\left[-(k-1)^{2} \varphi^{2} \alpha_{k-1}^{\prime 2}-k^{4} \eta^{2} \alpha_{k}^{\prime 2}+(k+1)^{2} \varphi^{2} \alpha_{k+1}^{\prime 2}\right]\right. \\
& \left.+2 \varphi^{2}\left[\alpha_{k-1}^{\prime 2}+\alpha_{k-1}^{\prime 2}\right] 2 \varphi^{\prime 2}\left[(k-1)^{2} \alpha_{k-1}^{2}+(k+1)^{2} \alpha_{k-1}^{2}\right]+\varphi^{\prime 2}\left[\alpha_{k-1}^{2}+\alpha_{k-1}^{2}\right]\right)
\end{aligned}
$$

By re-indexing the terms in (A.18), we get

$$
\begin{aligned}
\sum_{k=1}^{\infty}\left|\alpha_{k}^{\prime \prime}(t)\right|^{2} & \leq 4 \sum_{k=1}^{\infty}\left[6 \varphi^{2}\left(k \alpha_{k}^{\prime}\right)^{2}+3 \eta^{2}\left(k^{2} \alpha_{k}^{\prime}\right)^{2}+4 \varphi^{2}\left(\alpha_{k}^{\prime}\right)^{2}+4 \varphi^{\prime 2}\left(k \alpha_{k}\right)^{2}+2 \varphi^{\prime 2}\left(\alpha_{k}\right)^{2}\right] \\
& \leq 4\left[10 \varphi^{2} \sum_{k=1}^{\infty}\left(k \alpha_{k}^{\prime}\right)^{2}+3 \eta^{2} \sum_{k=1}^{\infty}\left(k^{2} \alpha_{k}^{\prime}\right)^{2}+6 \varphi^{\prime 2} \sum_{k=1}^{\infty}\left(k \alpha_{k}\right)^{2}\right] \\
\left\|\alpha_{k}^{\prime \prime}(t)\right\|_{2}^{2} & \leq 4\left(10 \varphi^{2}+3 \eta^{2}\right)\left\|k^{2} \alpha_{k}^{\prime}\right\|_{2}^{2}+6 \varphi^{\prime 2}\left\|k \alpha_{k}\right\|_{2}^{2}
\end{aligned}
$$


Use monotonicity of integration to get

$$
\int_{0}^{\infty}\left\|\alpha_{k}^{\prime \prime}(t)\right\|_{2}^{2} \mathrm{~d} t \leq \int_{0}^{\infty}\left(40 \varphi^{2}(t)\left\|k^{2} \alpha_{k}^{\prime}(t)\right\|_{2}^{2}+12 \eta^{2}\left\|k^{2} \alpha_{k}^{\prime}(t)\right\|_{2}^{2}+6 \varphi^{\prime 2}(t)\left\|k \alpha_{k}(t)\right\|_{2}^{2}\right) \mathrm{d} t
$$

Considering Lemma 3.4, we have

$$
6 \int_{0}^{\infty} \varphi^{\prime 2}(t)\left\|k \alpha_{k}(t)\right\|_{2}^{2} \mathrm{~d} t \leq \frac{12}{\left(\eta+c_{2}\right)}\left(\frac{\eta \pi u_{0} c_{1}}{L}\right)^{2}\|\widehat{\theta}(0)\|_{2}^{2}
$$

and using (A.16), we get

$$
40 \int_{0}^{\infty} \varphi^{2}(t)\left\|k^{2} \alpha_{k}^{\prime}(t)\right\|_{2}^{2} \mathrm{~d} t \leq \frac{14400 \pi^{4}\left|\bar{u}_{0}\right|^{4} a_{1}^{2}}{16 L^{4}\left(4 \eta+2 a_{2}\right)}\|\widehat{\beta}(0)\|_{2}^{2}+\frac{240 \pi^{2} \eta^{2}\left|\bar{u}_{0}\right|^{2} \lambda_{1}^{2}}{4 L^{2}(2(\eta+\lambda))}\|\widehat{\lambda}(0)\|_{2}^{2},
$$

and

$$
12 \eta^{2} \int_{0}^{\infty}\left\|k^{2} \alpha_{k}^{\prime}(t)\right\|_{2}^{2} \mathrm{~d} t \leq\left(\frac{540 \pi^{2}\left|\bar{u}_{0}\right|^{2} a_{1}^{2} \eta^{2}}{L^{2}\left(\eta+a_{2}\right)}\|\widehat{\beta}(0)\|_{2}^{2}+\frac{36 \eta^{4} \lambda_{1}^{2}}{\lambda_{2}}\|\widehat{\lambda}(0)\|_{2}^{2}\right)
$$

Putting it all together,

$$
\int_{0}^{\infty} \int_{0}^{L} A_{t t}^{2}(x, t) \mathrm{d} x \mathrm{~d} t \leq \xi_{1}\|\widehat{\theta}(0)\|_{2}^{2}+\xi_{2}\|\widehat{\beta}(0)\|_{2}^{2}+\xi_{3}\|\widehat{\lambda}(0)\|_{2}^{2}
$$

where $\xi_{1}=\frac{12}{\left(\eta+c_{2}\right)}\left(\frac{\eta \pi u_{0} c_{1}}{L}\right)^{2}, \xi_{2}=\frac{900 \pi^{4}\left|\bar{u}_{0}\right|^{4} a_{1}^{2}}{L^{4}\left(4 \eta+2 a_{2}\right)}+\frac{540 \pi^{2}\left|\bar{u}_{0}\right|^{2} a_{1}^{2} \eta^{2}}{L^{2}\left(\eta+a_{2}\right)}$, and

$\xi_{3}=\frac{30 \pi^{2} \eta^{2}\left|\bar{u}_{0}\right|^{2} \lambda_{1}^{2}}{L^{2}(\eta+\lambda)}+\frac{36 \eta^{4} \lambda_{1}^{2}}{\lambda_{2}}$.

\section{Appendix B. Main Results}

\section{B.1 Proof of Lemma 3.8}

Proof. Consider $v(x, y, t)=A(x, t)-\frac{\pi}{L} u_{0}(t) \cos \left(\frac{\pi x}{L}\right) y$. For all $(x, y) \in D(t)$, we have

$$
|v(x, y, t)| \leq|A(x, t)|+\frac{\pi}{L}\left|u_{0}(t)\right||f(x, t)|=\widetilde{v}(x, t) .
$$

We are assuming $f(x, t) \geq 0$. So, $f(x, t)=|f(x, t)|$. Now,

$$
f(x, t)=f_{i}(x)+\int_{0}^{t}\left(v(x, f(x, s), s)-\frac{\partial f(x, s)}{\partial x} u(x, s)\right) \mathrm{d} s,
$$

where $u(x, t)=u_{0}(t) \sin \left(\frac{\pi x}{L}\right)$. We also have, $f_{i}(x)=f(x, 0)=f\left(x_{0}\right)$. The capillary equation yields bounds on $\frac{\partial f(x, t)}{\partial x}$. By (2.10) and the boundary conditions, $\left|\frac{\partial f(x, t)}{\partial x}\right|$ is upper bounded by $C:=$ $\max \left\{\left|\cot \left(\theta_{1}(0)\right)\right|,\left|\cot \left(\theta_{1}(\infty)\right)\right|,\left|\cot \left(\theta_{2}(0)\right)\right|\right.$,

$\left.\left|\cot \left(\theta_{2}(\infty)\right)\right|\right\}$. Putting it all together:

$$
|\widetilde{v}(x, t)| \leq|A(x, t)|+\frac{\pi}{L}\left|u_{0}(t)\right|\left|f_{i}(x)\right|+\frac{\pi}{L}\left|u_{0}(t)\right| \int_{0}^{t}\left(|\widetilde{v}(x, s)|+C\left|u_{0}(s)\right|\right) \mathrm{d} s .
$$


Let

$$
\phi(x, t):=|A(x, t)|+\frac{\pi}{L}\left|u_{0}(t)\right|\left|f_{i}(x)\right|+\frac{\pi}{L}\left|u_{0}(t)\right| \int_{0}^{t} C\left|u_{0}(s)\right| \mathrm{d} s
$$

Then,

$$
|\widetilde{v}(x, t)| \leq \phi(x, t)+\frac{\pi}{L}\left|u_{0}(t)\right| \int_{0}^{t}|\widetilde{v}(x, s)| \mathrm{d} s .
$$

By (B.5) and the Gronwall-Bellman inequality [15, 17],

$$
|\widetilde{v}(x, t)| \leq \phi(x, t)+\frac{\pi}{L}\left|u_{0}(t)\right| \int_{0}^{t} \phi(x, s) e^{\frac{\pi}{L}\left|u_{0}(t)\right|(t-s)} \mathrm{d} s .
$$

Recall that $u_{0}(t)=e^{-\eta t} \bar{u}_{0}$ and $\eta=\frac{\mu \pi^{2}}{\rho L^{2}}$, and let $\omega=\frac{\pi}{L}\left|\bar{u}_{0}\right|$. Then,

$$
\begin{aligned}
|\widetilde{v}(x, t)| & \leq \phi(x, t)+\frac{\pi}{L} e^{-\eta t}\left|\bar{u}_{0}\right| \int_{0}^{t} \phi(x, s) e^{\frac{\pi\left|\bar{u}_{0}\right|}{L} e^{-\eta t}(t-s)} \mathrm{d} s \\
& \leq \phi(x, t)+\omega e^{-\left(\eta-\omega e^{-\eta t}\right) t} \int_{0}^{t} \phi(x, s) e^{-\omega s} \mathrm{~d} s .
\end{aligned}
$$

Since,

$$
\begin{aligned}
\phi(x, t) & =|A(x, t)|+\frac{\pi}{L}\left|u_{0}(t)\right|\left|f_{i}(x)\right|+\frac{\pi}{L}\left|u_{0}(t)\right| \int_{0}^{t} C\left|u_{0}(\tau)\right| \mathrm{d} \tau \\
& =|A(x, t)|+\frac{\pi}{L} e^{-\eta t}\left|\bar{u}_{0}\right|\left|f_{i}(x)\right|+\frac{\pi}{L}\left|\bar{u}_{0}\right| e^{-\eta t} C \int_{0}^{t} e^{-\eta \tau}\left|\bar{u}_{0}\right| \mathrm{d} \tau \\
\phi(x, t) & \leq|A(x, t)|+\left(\omega\left|f_{i}(x)\right|+\frac{\omega C\left|\bar{u}_{0}\right|^{2}}{\eta}\right) e^{-\eta t} .
\end{aligned}
$$

Now, consider $\int_{0}^{t} \phi(x, s) e^{-\omega s} \mathrm{~d} s$. By using the monotonicity of integration, we have

$$
\int_{0}^{t} \phi(x, s) e^{-\omega s} \mathrm{~d} s \leq \int_{0}^{t} \phi(x, s) \mathrm{d} s
$$

Hence, we focus on $\int_{0}^{t} \phi(x, s) \mathrm{d} s$.

$$
\begin{aligned}
\int_{0}^{t} \phi(x, s) \mathrm{d} s & \leq \int_{0}^{t}|A(x, s)| \mathrm{d} s+\left(\omega\left|f_{i}(x)\right|+\frac{\omega C\left|\bar{u}_{0}\right|^{2}}{\eta}\right) \int_{0}^{t} e^{-\eta s} \mathrm{~d} s \\
& =\int_{0}^{t}|A(x, s)| \mathrm{d} s+\frac{1}{\eta}\left(\omega\left|f_{i}(x)\right|+\frac{\omega C\left|\bar{u}_{0}\right|^{2}}{\eta}\right)\left(1-e^{-\eta t}\right) \\
& \leq \int_{0}^{t}|A(x, s)| \mathrm{d} s+\frac{1}{\eta}\left(\omega\left|f_{i}(x)\right|+\frac{\omega C\left|\bar{u}_{0}\right|^{2}}{\eta}\right)
\end{aligned}
$$


By (B.7) and (B.8),

$$
|\widetilde{v}(x, t)| \leq \phi(x, t)+\omega e^{-\left(\eta-\omega e^{-\eta t}\right) t}\left[\int_{0}^{t}|A(x, s)| \mathrm{d} s+\frac{\omega}{\eta}\left(\left|f_{i}(x)\right|+\frac{C\left|\bar{u}_{0}\right|^{2}}{\eta}\right)\right]
$$

Let $\sigma(x):=\frac{\omega}{\eta}\left(\left|f_{i}(x)\right|+\frac{C\left|\bar{u}_{0}\right|^{2}}{\eta}\right)$ and $\|\sigma\|:=\sup _{0 \leq x \leq L}|\sigma(x)|$.

$$
\int_{0}^{\infty} \int_{0}^{L}|\widetilde{v}(x, t)|^{2} \mathrm{~d} x \mathrm{~d} t \leq 3 \int_{0}^{\infty} \int_{0}^{L}\left(\phi^{2}(x, t)+\omega^{2} e^{-2\left(\eta-\omega e^{-\eta t}\right) t}\left(\int_{0}^{t}|A(x, s)| \mathrm{d} s\right)^{2}+\sigma^{2}(x) e^{-2\left(\eta-\omega e^{-\eta t}\right) t}\right) \mathrm{d} x \mathrm{~d} t
$$

Using (A.7), the integral

$$
\begin{aligned}
\int_{0}^{\infty} \int_{0}^{L} \phi^{2}(x, t) \mathrm{d} x \mathrm{~d} t & \leq 3 \int_{0}^{\infty} \int_{0}^{L}\left(A^{2}(x, t)+\omega^{2}\left|f_{i}(x)\right|^{2} e^{-2 \eta t}+\frac{\omega^{2} \mathrm{C}^{2}\left|\bar{u}_{0}\right|^{4}}{\eta^{2}} e^{-2 \eta t}\right) \mathrm{d} x \mathrm{~d} t \\
& \leq \frac{K_{1}^{2}}{2 K_{2}}\|\widehat{\alpha}(0)\|_{2}^{2}+\frac{\omega^{2}\left\|f_{i}\right\|^{2} L}{2 \eta}+\frac{\omega^{2} \mathrm{C}^{2}\left|\bar{u}_{0}\right|^{4}}{2 \eta^{3}}
\end{aligned}
$$

where $\left\|f_{i}\right\|:=\sup _{0 \leq x \leq L}\left|f_{i}(x)\right|$. The third integral in (B.10)

$$
\int_{0}^{\infty} \int_{0}^{L} \sigma^{2}(x) e^{-2\left(\eta-\omega e^{-\eta t}\right) t} \mathrm{~d} x \mathrm{~d} t \leq\|\sigma\|^{2} L \int_{0}^{\infty} e^{-2\left(\eta-\omega e^{-\eta t}\right) t} \mathrm{~d} t
$$

has following two possible cases.

Case 1: $\omega \geq \eta ; 0<\epsilon<\eta$. Let $t^{\star} \in(0, \infty) \ni \eta-\omega e^{-\eta t^{\star}}=\epsilon$. Note that for $t \in\left(t^{\star}, \infty\right), \eta-\omega e^{-\eta t}<\epsilon$. Therefore,

$$
\begin{aligned}
\int_{0}^{\infty} e^{-2\left(\eta-\omega e^{-\eta t}\right) t} \mathrm{~d} t & \leq \int_{0}^{t^{\star}} e^{-2\left(\eta-\omega e^{-\eta t}\right) t} \mathrm{~d} t+\int_{0}^{\infty} e^{-2 \epsilon t} \mathrm{~d} t \\
& \leq \int_{0}^{t^{\star}} e^{2(\omega-\eta) t} \mathrm{~d} t+\int_{0}^{\infty} e^{-2 \epsilon t} \mathrm{~d} t=\Omega+\frac{1}{2 \epsilon} \leq \Omega+\frac{1}{\eta} .
\end{aligned}
$$

where $\Omega=\int_{0}^{t^{\star}} e^{2(\omega-\eta) t} \mathrm{~d} t$, a constant, and we pick $\epsilon=\frac{\eta}{2}$.

Case 2: $\omega<\eta \forall t \geq 0$. Then

$$
\int_{0}^{\infty} e^{-2\left(\eta-\omega e^{-\eta t}\right) t} \mathrm{~d} t \leq \int_{0}^{\infty} e^{-2(\eta-\omega) t} \mathrm{~d} t=\frac{1}{2(\eta-\omega)}<\frac{1}{\eta}
$$

Therefore, equation (B.12)

$$
\int_{0}^{\infty} \int_{0}^{L} \sigma^{2} e^{-2\left(\eta-\omega e^{-\eta t}\right) t} \mathrm{~d} x \mathrm{~d} t \leq \begin{cases}\|\sigma\|^{2} L\left(\Omega+\frac{1}{\eta}\right) & \text { if } \omega \geq \eta \\ \frac{\|\sigma\|^{2} L}{\eta} & \text { if } \omega<\eta\end{cases}
$$


Finally, we focus on the second integral in (B.10)

$$
\int_{0}^{\infty} \int_{0}^{L} \omega^{2} e^{-2\left(\eta-\omega e^{-\eta t}\right) t}\left(\int_{0}^{t}|A(x, s)| \mathrm{d} s\right)^{2} \mathrm{~d} x \mathrm{~d} t
$$

We use the Cauchy-Schwarz inequality to get: $\left(\int_{0}^{t}|A(x, s)| \mathrm{d} s\right)^{2} \leq t \int_{0}^{t}|A(x, s)|^{2} \mathrm{~d} s$. Thus,

$$
\int_{0}^{\infty} \int_{0}^{L} \omega^{2} e^{-2\left(\eta-\omega e^{-\eta t}\right) t}\left(\int_{0}^{t}|A(x, s)| \mathrm{d} s\right)^{2} \mathrm{~d} x \mathrm{~d} t \leq \int_{0}^{\infty} \int_{0}^{L} \omega^{2} e^{-2\left(\eta-\omega e^{-\eta t}\right) t} t \int_{0}^{t}|A(x, s)|^{2} \mathrm{~d} s \mathrm{~d} x \mathrm{~d} t .
$$

Consider,

$$
\int_{0}^{\infty} \omega^{2} e^{-2\left(\eta-\omega e^{-\eta t}\right) t} t \int_{0}^{t} \int_{0}^{L}|A(x, s)|^{2} \mathrm{~d} x \mathrm{~d} s \mathrm{~d} t
$$

By Parserval-Plancherel theorem,

$$
\begin{aligned}
\int_{0}^{\infty} \omega^{2} e^{-2\left(\eta-\omega e^{-\eta t}\right) t} t \int_{0}^{t} \int_{0}^{L}|A(x, s)|^{2} \mathrm{~d} x \mathrm{~d} s \mathrm{~d} t & =\int_{0}^{\infty} \omega^{2} e^{-2\left(\eta-\omega e^{-\eta t}\right) t} t \int_{0}^{t}\|\alpha(s)\|_{2}^{2} \mathrm{~d} s \mathrm{~d} t \\
& \leq \omega^{2} \int_{0}^{\infty} e^{-2\left(\eta-\omega e^{-\eta t}\right) t} t \int_{0}^{t} K_{1}^{2} e^{-2 K_{2} s}\|\widehat{\alpha}(0)\|_{2}^{2} \mathrm{~d} s \mathrm{~d} t \\
& =\frac{\omega^{2} K_{1}^{2}\|\widehat{\alpha}(0)\|_{2}^{2}}{2 K_{2}} \int_{0}^{\infty} e^{-2\left(\eta-\omega e^{-\eta t}\right) t} t\left(1-e^{-2 K_{2} t}\right) \mathrm{d} t \\
& \leq \frac{\omega^{2} K_{1}^{2}\|\widehat{\alpha}(0)\|_{2}^{2}}{2 K_{2}} \int_{0}^{\infty} t e^{-2\left(\eta-\omega e^{-\eta t}\right) t} \mathrm{~d} t .
\end{aligned}
$$

Case 1: $\omega<\eta$

$$
\begin{aligned}
\int_{0}^{\infty} t e^{-2\left(\eta-\omega e^{-\eta t}\right) t} \mathrm{~d} t & \leq \int_{0}^{\infty} t e^{-2(\eta-\omega) t} \mathrm{~d} t \\
& =\lim _{N \rightarrow \infty}\left[\left.\frac{t e^{-2(\eta-\omega) t}}{-2(\eta-\omega)}\right|_{0} ^{N}+\frac{1}{2(\eta-\omega)} \int_{0}^{N} e^{-2(\eta-\omega) t} \mathrm{~d} t\right] \\
& =\frac{1}{4(\eta-\omega)^{2}}<\frac{1}{\eta^{2}} .
\end{aligned}
$$

Case 2: $\omega \geq \eta$

$$
\begin{aligned}
\int_{0}^{\infty} t e^{-2\left(\eta-\omega e^{-\eta t}\right) t} \mathrm{~d} t & \leq \int_{0}^{t^{\star}} t e^{-2\left(\eta-\omega e^{-\eta t}\right) t} \mathrm{~d} t+\int_{0}^{\infty} t e^{-2\left(\eta-\omega e^{-\eta t}\right) t} \mathrm{~d} t \\
& \leq \int_{0}^{t^{\star}} t e^{2(\omega-\eta) t} \mathrm{~d} t+\int_{0}^{\infty} t e^{-2 \epsilon t} \mathrm{~d} t \\
& =\left[\frac{t^{\star} e^{2(\omega-\eta) t^{\star}}}{2(\omega-\eta)}+\frac{\left(1-e^{2(\omega-\eta) t^{\star}}\right)}{4(\omega-\eta)^{2}}\right]+\frac{1}{4 \epsilon^{2}}<\widehat{\Omega}+\frac{1}{\eta^{2}},
\end{aligned}
$$


where $\widehat{\Omega}=\left[\frac{t^{\star} e^{2 \omega t^{\star}}}{2 \omega}+\frac{1}{4 \omega^{2}}\right]$. Hence,

$$
\int_{0}^{\infty} \int_{0}^{L} \omega^{2} e^{-2\left(\eta-\omega e^{-\eta t}\right) t}\left(\int_{0}^{t}|A(x, s)| \mathrm{d} s\right)^{2} \mathrm{~d} x \mathrm{~d} t \leq\left\{\begin{array}{l}
\frac{\omega^{2} K_{1}^{2}\|\widehat{\alpha}(0)\|_{2}^{2}}{2 K_{2}}\left(\widehat{\Omega}+\frac{1}{\eta^{2}}\right) \text { if } \omega \geq \eta \\
\frac{\omega^{2} K_{1}^{2}\|\widehat{\alpha}(0)\|_{2}^{2}}{8 K_{2} \eta^{2}} \text { if } \omega<\eta .
\end{array}\right.
$$

Thus, using (B.10), (B.11), (B.15), and (B.18), we have

$$
\int_{0}^{\infty} \int_{0}^{L}|\widetilde{v}(x, t)|^{2} \mathrm{~d} x \mathrm{~d} t \leq \begin{cases}\frac{K_{1}^{2}\|\widehat{\alpha}(0)\|_{2}^{2}}{2 K_{2}}\left(1+\omega^{2}\left(\widehat{\Omega}+\frac{1}{\eta^{2}}\right)\right)+L\|\sigma\|^{2}\left(\Omega+\frac{1}{\eta}\right) & \text { if } \omega \geq \eta \\ \frac{K_{1}^{2}\|\widehat{\alpha}(0)\|_{2}^{2}}{2 K_{2}}\left(1+\frac{\omega^{2}}{4 \eta^{2}}\right)+\frac{L\|\sigma\|^{2}}{\eta} & \text { if } \omega<\eta\end{cases}
$$

Claim: The integral

$$
\int_{0}^{\infty} \int_{0}^{L} e^{-2 \eta, t}|f(x, t)|^{2} \mathrm{~d} x \mathrm{~d} t \leq\left\{\begin{array}{l}
\frac{3 L}{2 \eta}\left(\left\|f_{i}\right\|^{2}+\frac{\mathrm{C}^{2}\left|\bar{u}_{0}\right|^{2}}{\eta^{2}}\right)+\frac{9 K_{1}^{2}\|\widehat{\alpha}(0)\|_{2}^{2}}{2 \eta^{3}}+\frac{3\|\widehat{\sigma}\|_{2}^{2}}{\eta^{3}}\left[1+\frac{3 \omega^{2}}{2}(\eta(2 \widehat{\Omega}+\Omega)+3)\right] \\
+\frac{1}{4 \eta^{4}}(2 \widetilde{\Omega}+\widehat{\Omega}+5) \text { if } \omega \geq \eta \\
\frac{3 L}{2 \eta}\left(\left\|f_{i}\right\|^{2}+\frac{\mathrm{C}^{2}\left|\bar{u}_{0}\right|^{2}}{\eta^{2}}\right)+\frac{9 K_{1}^{2}\|\widehat{\alpha}(0)\|_{2}^{2}}{2 \eta^{3}}+\frac{3\|\widehat{\sigma}\|_{2}^{2}}{\eta^{3}}\left(1+2 \omega^{2}\right)+\frac{2}{\eta^{4}} \text { if } \omega<\eta .
\end{array}\right.
$$

Proof.

$$
\int_{0}^{\infty} \int_{0}^{L} e^{-2 \eta, t}|f(x, t)|^{2} \mathrm{~d} x \mathrm{~d} t \leq \int_{0}^{\infty} \int_{0}^{L} e^{-2 \eta, t}\left[\left|f_{i}(x)\right|+\int_{0}^{t}\left(|v(x, f(x, s), s)|+\left|\frac{\partial f(x, s)}{\partial x}\right||u(x, s)|\right) \mathrm{d} s\right]^{2} \mathrm{~d} x \mathrm{~d} t
$$

Thus,

$$
\begin{aligned}
& \leq \int_{0}^{\infty} \int_{0}^{L} e^{-2 \eta t}\left[\left|f_{i}(x)\right|+\int_{0}^{t}\left(|v(x, f(x, s), s)|+\left|\frac{\partial f(x, s)}{\partial x}\right||u(x, s)|\right) \mathrm{d} s\right]^{2} \mathrm{~d} x \mathrm{~d} t \\
& \leq 3 \int_{0}^{\infty} \int_{0}^{L} e^{-2 \eta t}\left(\left(\left|f_{i}(x)\right|^{2}+\frac{\mathrm{C}^{2}\left|\bar{u}_{0}^{2}\right|}{\eta^{2}}\right)+\left[\int_{0}^{t}|v(x, f(x, s), s)| \mathrm{d} s\right]^{2}\right) \mathrm{d} x \mathrm{~d} t
\end{aligned}
$$

Observe that $\left[\int_{0}^{t}|v(x, f(x, s), s)| \mathrm{d} s\right]^{2} \leq t \int_{0}^{t}|v(x, f(x, s), s)|^{2} \mathrm{~d} s$. Therefore, the last integral in (B.22) can be bounded from above by

$$
3 \int_{0}^{\infty} \int_{0}^{L} t e^{-2 \eta t}\left(\int_{0}^{t}|v(x, f(x, s), s)|^{2} \mathrm{~d} s\right) \mathrm{d} x \mathrm{~d} t
$$


We use $\int_{0}^{t}|v(x, f(x, s), s)|^{2} \mathrm{~d} s \leq \int_{0}^{t}|\widetilde{v}(x, s)|^{2} \mathrm{~d} s$ and (B.9) to get

$$
\begin{aligned}
& \int_{0}^{t}|v(x, f(x, s), s)|^{2} \mathrm{~d} s \\
& \leq 3 \int_{0}^{t}\left[\phi^{2}(x, s)+2 \omega^{2} e^{-2\left(\eta-\omega e^{-\eta s}\right) s}\left(\sigma^{2}(x)+\left[\int_{0}^{s}|A(x, \tau)| \mathrm{d} \tau\right]^{2}\right)\right] \mathrm{d} s \\
& \leq 3 \int_{0}^{t} \phi^{2}(x, s) \mathrm{d} s+6 \omega^{2} \sigma^{2}(x) \int_{0}^{t} e^{-2\left(\eta-\omega e^{-\eta s}\right) s} \mathrm{~d} s \\
& \quad+6 \omega^{2} \int_{0}^{t}\left(s e^{-2\left(\eta-\omega e^{-\eta s}\right) s} \int_{0}^{s}|A(x, \tau)|^{2} \mathrm{~d} \tau\right) \mathrm{d} s .
\end{aligned}
$$

By the definition of $\phi($ see (B.5)), we get

$$
\phi(x, s) \leq|A(x, s)|+\left(\frac{\pi\left|\bar{u}_{0}\right|}{L}\left|f_{i}(x)\right|+\frac{\pi\left|\bar{u}_{0}\right| C}{L \eta}\right) e^{-\eta s}=|A(x, s)|+\widehat{\sigma}(x) e^{-\eta s}
$$

where $\widehat{\sigma}(x)=\left(\frac{\pi\left|\bar{u}_{0}\right|}{L}\left|f_{i}(x)\right|+\frac{\pi\left|\bar{u}_{0}\right| C}{L \eta}\right)$. Let $\|\widehat{\sigma}\|:=\sup _{0 \leq x \leq L}|\widehat{\sigma}(x)|$. Consider the first integral in (B.24):

$$
\int_{0}^{t} \phi^{2}(x, s) \mathrm{d} s \leq 2 \int_{0}^{t}|A(x, s)|^{2} \mathrm{~d} s+2 \int_{0}^{t} \widehat{\sigma}^{2}(x) e^{-2 \eta s} \mathrm{~d} s \leq 2 \int_{0}^{t}|A(x, s)|^{2} \mathrm{~d} s+\frac{\widehat{\sigma}^{2}(x)}{\eta} .
$$

We use (B.23), (B.24), and (B.25) to obtain

$$
\begin{aligned}
& \int_{0}^{\infty} \int_{0}^{L} t e^{-2 \eta t}\left(\int_{0}^{t}|v(x, f(x, s), s)|^{2} \mathrm{~d} s\right) \mathrm{d} x \mathrm{~d} t \\
& \leq \int_{0}^{\infty} \int_{0}^{L} t e^{-2 \eta t}\left[6 \int_{0}^{t}|A(x, s)|^{2} \mathrm{~d} s+\frac{3 \widehat{\sigma}^{2}(x)}{\eta}+6 \omega^{2} \sigma^{2}(x) \int_{0}^{t} e^{-2\left(\eta-\omega e^{-\eta s}\right) s} \mathrm{~d} s\right. \\
& \left.\quad+6 \omega^{2} \int_{0}^{t}\left(s e^{-2\left(\eta-\omega e^{-\eta s}\right) s} \int_{0}^{s}|A(x, \tau)|^{2} \mathrm{~d} \tau\right) \mathrm{d} s\right] \mathrm{d} x \mathrm{~d} t .
\end{aligned}
$$

Consider,

$$
\begin{aligned}
6 \int_{0}^{\infty} \int_{0}^{t} t e^{-2 \eta t} \int_{0}^{L}|A(x, s)|^{2} \mathrm{~d} x \mathrm{~d} s \mathrm{~d} t & =6 \int_{0}^{\infty} \int_{0}^{t} t e^{-2 \eta t}\|\widehat{\alpha}(s)\|_{2}^{2} \mathrm{~d} s \mathrm{~d} t \\
& \leq 6 \int_{0}^{\infty} t e^{-2 \eta t} \int_{0}^{t} K_{1}^{2} e^{-2 K_{2} s}\|\widehat{\alpha}(0)\|_{2}^{2} \mathrm{~d} s \mathrm{~d} t \\
& \leq 6 K_{1}^{2}\|\widehat{\alpha}(0)\|_{2}^{2} \int_{0}^{\infty} t^{2} e^{-2 \eta t} \mathrm{~d} t=\frac{6 K_{1}^{2}\|\widehat{\alpha}(0)\|_{2}^{2}}{4 \eta^{3}}
\end{aligned}
$$

Further, the second integral in (B.26) can be upper bounded by

$$
\frac{3}{\eta} \int_{0}^{\infty} \int_{0}^{L} t e^{-2 \eta t} \widehat{\sigma}^{2}(x) \mathrm{d} x \mathrm{~d} t=\frac{3}{4 \eta^{3}}\|\widehat{\sigma}\|_{2}^{2}<\frac{\|\widehat{\sigma}\|_{2}^{2}}{\eta^{3}}
$$


The integral:

$$
\begin{aligned}
6 & \omega^{2} \int_{0}^{\infty} \int_{0}^{L} t e^{-2 \eta t} \sigma^{2}(x) \int_{0}^{t} e^{-2\left(\eta-\omega e^{-\eta s}\right) s} \mathrm{~d} s \mathrm{~d} x \mathrm{~d} t \\
& =6 \omega^{2}\|\widehat{\sigma}\|_{2}^{2} \int_{0}^{\infty} t e^{-2 \eta t} \int_{0}^{t} e^{-2\left(\eta-\omega e^{-\eta s}\right) s} \mathrm{~d} s \mathrm{~d} t \\
& =6 \omega^{2}\|\widehat{\sigma}\|_{2}^{2} \int_{0}^{\infty} e^{-2\left(\eta-\omega e^{-\eta s}\right) s} \int_{t=s}^{\infty} t e^{-2 \eta t} \mathrm{~d} t \mathrm{~d} s \\
& \leq 6 \omega^{2}\|\widehat{\sigma}\|_{2}^{2}\left[\frac{1}{2 \eta} \int_{0}^{\infty} s e^{-2\left(\eta-\omega e^{-\eta s}\right) s} \mathrm{~d} s+\frac{1}{4 \eta^{2}} \int_{0}^{\infty} e^{-2\left(\eta-\omega e^{-\eta s}\right) s} \mathrm{~d} s\right]
\end{aligned}
$$

By considering the results in (B.13), (B.14), (B.16), and (B.17); one can get

$$
\begin{aligned}
& 6 \omega^{2} \int_{0}^{\infty} \int_{0}^{L} t e^{-2 \eta t} \sigma^{2}(x) \int_{0}^{t} e^{-2\left(\eta-\omega e^{-\eta s}\right) s} \mathrm{~d} s \mathrm{~d} x \mathrm{~d} t \\
& \quad \leq \begin{cases}6 \omega^{2}\|\widehat{\sigma}\|_{2}^{2}\left[\frac{1}{2 \eta}\left(\widehat{\Omega}+\frac{1}{\eta^{2}}\right)+\frac{1}{4 \eta^{2}}\left(\Omega+\frac{1}{\eta}\right)\right] & \text { if } \omega \geq \eta \\
6 \omega^{2}\|\widehat{\sigma}\|_{2}^{2}\left[\frac{1}{2 \eta^{3}}+\frac{1}{4 \eta^{3}}\right] & \text { if } \omega<\eta .\end{cases}
\end{aligned}
$$

Now we focus on the integral:

$$
6 \omega^{2} \int_{0}^{\infty} \int_{0}^{L} t e^{-2 \eta t} \int_{0}^{t}\left(s e^{-2\left(\eta-\omega e^{-\eta s}\right) s} \int_{0}^{s}|A(x, \tau)|^{2} \mathrm{~d} \tau\right) \mathrm{d} s \mathrm{~d} x \mathrm{~d} t .
$$

Consider,

$$
\begin{aligned}
& \int_{0}^{\infty} t e^{-2 \eta t} \int_{0}^{t}\left(s e^{-2\left(\eta-\omega e^{-\eta s}\right) s} \int_{0}^{s} \int_{0}^{L}|A(x, \tau)|^{2} \mathrm{~d} x \mathrm{~d} \tau\right) \mathrm{d} s \mathrm{~d} t \\
& \quad=\int_{0}^{\infty} t e^{-2 \eta t} \int_{0}^{t} s e^{-2\left(\eta-\omega e^{-\eta s}\right) s} \int_{0}^{s}\|\alpha(\tau)\|_{2}^{2} \mathrm{~d} \tau \mathrm{d} s \mathrm{~d} t \\
& \leq \int_{0}^{\infty} t e^{-2 \eta t} \int_{0}^{t} s e^{-2\left(\eta-\omega e^{-\eta s}\right) s} \int_{0}^{s} K_{1}^{2} e^{-2 K_{2} \tau}\|\widehat{\alpha}(0)\|_{2}^{2} \mathrm{~d} \tau \mathrm{d} s \mathrm{~d} t \\
& \leq \frac{K_{1}^{2}\|\widehat{\alpha}(0)\|_{2}^{2}}{2 K_{2}} \int_{0}^{\infty} t e^{-2 \eta t} \int_{0}^{t} s e^{-2\left(\eta-\omega e^{-\eta s}\right) s} \mathrm{~d} s \mathrm{~d} t \\
& =\frac{K_{1}^{2}\|\widehat{\alpha}(0)\|_{2}^{2}}{2 K_{2}} \int_{0}^{\infty} s e^{-2\left(\eta-\omega e^{-\eta s}\right) s} \int_{t=s}^{\infty} t e^{-2 \eta t} \mathrm{~d} t \mathrm{~d} s
\end{aligned}
$$

Hence,

$$
\begin{aligned}
\int_{0}^{\infty} s e^{-2\left(\eta-\omega e^{-\eta s}\right) s} \int_{t=s}^{\infty} t e^{-2 \eta t} \mathrm{~d} t \mathrm{~d} s & =\int_{0}^{\infty} s e^{-2\left(\eta-\omega e^{-\eta s}\right) s}\left(\frac{e^{-2 \eta s}}{4 \eta^{2}}+\frac{s e^{-2 \eta s}}{2 \eta}\right) \mathrm{d} s \\
& \leq \int_{0}^{\infty}\left(\frac{s}{4 \eta^{2}}+\frac{s^{2}}{2 \eta}\right) e^{-2\left(\eta-\omega e^{-\eta s}\right) s} \mathrm{~d} s
\end{aligned}
$$

Consider: $\int_{0}^{\infty} s^{2} e^{-2\left(\eta-\omega e^{-\eta s}\right) s} \mathrm{~d} s$ 
Case 1: $\omega \geq \eta$;

Let $0<\epsilon<\eta$ and $t^{\star} \in(0, \infty) \ni \eta-\omega e^{-\eta t^{\star}}=\epsilon$. Note that for $t \in\left(t^{\star}, \infty\right), \eta-\omega e^{-\eta t}<\epsilon$.

$$
\begin{aligned}
\int_{0}^{\infty} s^{2} e^{-2\left(\eta-\omega e^{-\eta s}\right) s} \mathrm{~d} s & \leq \int_{0}^{t^{\star}} s^{2} e^{-2\left(\eta-\omega e^{-\eta s}\right) s} \mathrm{~d} s+\int_{0}^{t^{\star}} s^{2} e^{-2\left(\eta-\omega e^{-\eta s}\right) s} \mathrm{~d} s \\
& \leq \int_{0}^{t^{\star}} s^{2} e^{2(\omega-\eta) s} \mathrm{~d} s+\int_{0}^{\infty} s^{2} e^{-2 \epsilon s} \mathrm{~d} s=\widetilde{\Omega}+\frac{1}{4 \epsilon^{3}}
\end{aligned}
$$

where $\widetilde{\Omega}=\int_{0}^{t^{\star}} s^{2} e^{2(\omega-\eta) s} \mathrm{~d} s$. If we pick $\epsilon=\frac{\eta}{2}$,

$$
\int_{0}^{\infty} s^{2} e^{-2\left(\eta-\omega e^{-\eta s}\right) s} \mathrm{~d} s \leq \widetilde{\Omega}+\frac{2}{\eta^{3}}
$$

Case 2: $\omega<\eta$

$$
\int_{0}^{\infty} s^{2} e^{-2\left(\eta-\omega e^{-\eta s}\right) s} \mathrm{~d} s \leq \int_{0}^{\infty} s^{2} e^{-2(\eta-\omega) s} \mathrm{~d} s \leq \frac{1}{4(\eta-\omega)^{3}}<\frac{1}{\eta^{3}}
$$

Putting it all together:

$$
\int_{0}^{\infty} t e^{-2 \eta t} \int_{0}^{t} s e^{-2\left(\eta-\omega e^{-\eta s}\right) s} \mathrm{~d} s \mathrm{~d} t \leq\left\{\begin{array}{l}
{\left[\frac{1}{4 \eta^{2}}\left(\widehat{\Omega}+\frac{1}{\eta^{2}}\right)+\frac{1}{2 \eta}\left(\widetilde{\Omega}+\frac{2}{\eta^{3}}\right)\right] \text { if } \omega \geq \eta} \\
\left(\frac{1}{4 \eta^{4}}+\frac{1}{\eta^{4}}\right) \text { if } \omega<\eta
\end{array}\right.
$$

Therefore,

$$
\int_{0}^{\infty} \int_{0}^{L} e^{-2 \eta t}|f(x, t)|^{2} \mathrm{~d} x \mathrm{~d} t \leq\left\{\begin{array}{l}
\frac{3 L}{2 \eta}\left(\left\|f_{i}\right\|^{2}+\frac{\mathrm{C}^{2}\left|\bar{u}_{0}\right|^{2}}{\eta^{2}}\right)+\frac{9 K_{1}^{2}\|\widehat{\alpha}(0)\|_{2}^{2}}{2 \eta^{3}}+\frac{3\|\widehat{\sigma}\|_{2}^{2}}{\eta^{3}}\left[1+\frac{3 \omega^{2}}{2}(\eta(2 \widehat{\Omega}+\Omega)+3)\right] \\
+\frac{1}{4 \eta^{4}}(2 \widetilde{\Omega}+\widehat{\Omega}+5) \text { if } \omega \geq \eta \\
\frac{3 L}{2 \eta}\left(\left\|f_{i}\right\|^{2}+\frac{\mathrm{C}^{2}\left|\bar{u}_{0}\right|^{2}}{\eta^{2}}\right)+\frac{9 K_{1}^{2}\|\widehat{\alpha}(0)\|_{2}^{2}}{2 \eta^{3}}+\frac{3\|\widehat{\sigma}\|_{2}^{2}}{\eta^{3}}\left(1+2 \omega^{2}\right)+\frac{2}{\eta^{4}} \text { if } \omega<\eta .
\end{array}\right.
$$

Lemma B.1. $v_{t} \in L^{2}((0, L) \times(0, \infty))$

Proof. Since $v(x, y, t)=A(x, t)-\frac{\pi}{L} u_{0}(t) \cos \left(\frac{\pi x}{L}\right) y$ and $\frac{d(x, y)}{\mathrm{d} t}=(u(x, y, t), v(x, y, t))$, we have

$$
\begin{aligned}
v_{t} & =A_{t}+A_{x} u(x, t)-\frac{\pi}{L}\left[u_{0}^{\prime}(t) \cos \left(\frac{\pi x}{L}\right) y+u_{0}(t) \cos \left(\frac{\pi x}{L}\right) v(x, y, t)-\frac{\pi}{L} u_{0}(t) \sin \left(\frac{\pi x}{L}\right) y u(x, t)\right], \\
\left|v_{t}\right| & \leq\left|A_{t}\right|+\left|A_{x}\right|\left|\bar{u}_{0}\right| e^{-\eta t}+\frac{\pi}{L}\left[\eta\left|\bar{u}_{0}\right| e^{-\eta t} f(x, t)+\left|\bar{u}_{0}\right| e^{-\eta t}|v(x, y, t)|+\frac{\pi}{L}\left|\bar{u}_{0}\right|^{2} e^{-2 \eta t} f(x, t)\right]
\end{aligned}
$$

Thus,

$$
\begin{aligned}
\int_{0}^{\infty} \int_{0}^{L}\left|v_{t}\right|^{2} \mathrm{~d} x \mathrm{~d} t \leq & 5 \int_{0}^{\infty} \int_{0}^{L}\left|A_{t}\right|^{2} \mathrm{~d} x \mathrm{~d} t+5 \frac{\pi^{2}}{L^{4}}\left(\eta^{2} L^{2}+\pi^{2}\left|\bar{u}_{0}\right|^{2}\right) \int_{0}^{\infty} \int_{0}^{L} e^{-2 \eta t}|f(x, t)|^{2} \mathrm{~d} x \mathrm{~d} t \\
& +5\left|\bar{u}_{0}\right|^{2} \int_{0}^{\infty} \int_{0}^{L}\left|A_{x}\right|^{2} \mathrm{~d} x \mathrm{~d} t+\frac{5 \pi^{2}\left|\bar{u}_{0}\right|^{2}}{L^{2}} \int_{0}^{\infty} \int_{0}^{L}|v(x, y, t)|^{2} \mathrm{~d} x \mathrm{~d} t,
\end{aligned}
$$




$$
\begin{aligned}
\leq & 5 \int_{0}^{\infty} \int_{0}^{L}\left|A_{t}\right|^{2} \mathrm{~d} x \mathrm{~d} t+5 \frac{\pi^{2}}{L^{4}}\left(\eta^{2} L^{2}+\pi^{2}\left|\bar{u}_{0}\right|^{2}\right) \int_{0}^{\infty} \int_{0}^{L} e^{-2 \eta t}|f(x, t)|^{2} \mathrm{~d} x \mathrm{~d} t \\
& +5\left|\bar{u}_{0}\right|^{2} \int_{0}^{\infty} \int_{0}^{L}\left|A_{x}\right|^{2} \mathrm{~d} x \mathrm{~d} t+\frac{5 \pi^{2}\left|\bar{u}_{0}\right|^{2}}{L^{2}} \int_{0}^{\infty} \int_{0}^{L}|\widetilde{v}(x, t)|^{2} \mathrm{~d} x \mathrm{~d} t .
\end{aligned}
$$

Using (A.7), (A.8), (B.19), and (B.27), we have $v_{t} \in L^{2}([0, L] \times(0, \infty))$

Lemma B.2. $v_{t t} \in L^{2}([0, L] \times(0, \infty))$

Proof. Recall (B.28). Then,

$$
\begin{aligned}
v_{t t}= & A_{t t}+A_{t x} u(x, t)+u(x, t)\left[A_{x x} u(x, t)+A_{x t}\right]+A_{x}(x, t)\left[u_{x}(x, t) u(x, t)+u_{t}(x, t)\right] \\
& -\frac{\pi}{L}\left[u_{0}^{\prime \prime}(t) \cos \left(\frac{\pi x}{L}\right) y-\frac{\pi}{L} u_{0}^{\prime}(t) \sin \left(\frac{\pi x}{L}\right) u(x, t) y+u_{0}^{\prime}(t) \cos \left(\frac{\pi x}{L}\right) v(x, y, t)\right. \\
& +u_{0}^{\prime}(t) \cos \left(\frac{\pi x}{L}\right) v(x, y, t)-\frac{\pi}{L} u_{0}(t) \sin \left(\frac{\pi x}{L}\right) u(x, t) v(x, y, t) \\
& +u_{0}(t) \cos \left(\frac{\pi x}{L}\right) v_{t}(x, y, t)-\frac{\pi}{L} u_{0}^{\prime}(t) \sin \left(\frac{\pi x}{L}\right) y u(x, t)-\frac{\pi^{2}}{L^{2}} u_{0}(t) \cos \left(\frac{\pi x}{L}\right) y u^{2}(x, t) \\
& \left.-\frac{\pi}{L} u_{0}(t) \sin \left(\frac{\pi x}{L}\right) v(x, y, t) u(x, t)-\frac{\pi}{L} u_{0}(t) \sin \left(\frac{\pi x}{L}\right) y\left(u_{t}(x, t)+u_{x}(x, t) u(x, t)\right)\right] .
\end{aligned}
$$

Using $u(x, t)=\bar{u}_{0} e^{-\eta t} \sin \left(\frac{\pi x}{L}\right)$ and observing $|u(x, t)| \leq\left|\bar{u}_{0}\right|$, we get

$$
\begin{aligned}
\frac{L}{\pi}\left|v_{t t}\right| \leq & \frac{L}{\pi}\left(\left|A_{t t}\right|+2\left|\bar{u}_{0}\right|\left|A_{t x}\right|+\left|\bar{u}_{0}\right|^{2}\left|A_{x x}\right|+\left|\bar{u}_{0}\right|\left(\frac{\pi\left|\bar{u}_{0}\right|}{L}+\eta\right)\left|A_{x}\right|\right) \\
& +|f(x, t)|\left(\left|\bar{u}_{0}\right| \eta^{2} e^{-\eta t}+\frac{2 \pi \eta\left|\bar{u}_{0}\right|^{2}}{L} e^{-2 \eta t}+\frac{\pi^{2}\left|\bar{u}_{0}\right|^{3}}{L^{2}} e^{-3 \eta t}\right. \\
& \left.+\frac{\pi\left|\bar{u}_{0}\right|}{L} e^{-\eta t}\left(\eta\left|\bar{u}_{0}\right| e^{-\eta t}+\frac{\pi}{L}\left|\bar{u}_{0}\right|^{2} e^{-2 \eta t}\right)\right) \\
& +|\widetilde{v}(x, t)|\left(2 \eta\left|\bar{u}_{0}\right| e^{-\eta t}+\frac{2 \pi\left|\bar{u}_{0}\right|^{2}}{L} e^{-2 \eta t}\right)+\left|\bar{u}_{0}\right| e^{-\eta t}\left|v_{t}(x, y, t)\right| \\
\leq & \frac{L}{\pi}\left(\left|A_{t t}\right|+2\left|\bar{u}_{0}\right|\left|A_{t x}\right|+\left|\bar{u}_{0}\right|^{2}\left|A_{x x}\right|+\left|\bar{u}_{0}\right|\left(\frac{\pi}{L}\left|\bar{u}_{0}\right|+\eta\right)\left|A_{x}\right|\right) \\
& +|f(x, t)| e^{-\eta t}\left(\left|\bar{u}_{0}\right| \eta^{2}+\frac{3 \pi \eta\left|\bar{u}_{0}\right|^{2}}{L}+\frac{2 \pi^{2}\left|\bar{u}_{0}\right|^{3}}{L^{2}}\right)+\left|\bar{u}_{0}\right|\left|v_{t}(x, y, t)\right| \\
& +|\widetilde{v}(x, t)| e^{-\eta t}\left(2 \eta\left|\bar{u}_{0}\right|+\frac{2 \pi\left|\bar{u}_{0}\right|^{2}}{L}\right), \\
\left|v_{t t}\right| \leq & \left(\left|A_{t t}\right|+2\left|\bar{u}_{0}\right|\left|A_{t x}\right|+\left|\bar{u}_{0}\right|^{2}\left|A_{x x}\right|+\left|\bar{u}_{0}\right|\left(\frac{\pi\left|\bar{u}_{0}\right|}{L}+\eta\right)\left|A_{x}\right|\right)+\zeta_{1}|f(x, t)| e^{-\eta t} \\
& +\zeta_{2}\left|v_{t}\right|+\zeta_{3}|\widetilde{v}| e^{-\eta t},
\end{aligned}
$$

where $\zeta_{1}:=\frac{\pi}{L}\left(\left|\bar{u}_{0}\right| \eta^{2}+\frac{3 \pi \eta\left|\bar{u}_{0}\right|^{2}}{L}+\frac{2 \pi^{2}\left|\bar{u}_{0}\right|^{3}}{L^{2}}\right), \zeta_{2}:=\frac{\pi\left|\bar{u}_{0}\right|}{L}$, and $\zeta_{3}:=\frac{\pi}{L}\left(2 \eta\left|\bar{u}_{0}\right|+\frac{2 \pi\left|\bar{u}_{0}\right|^{2}}{L}\right)$. Therefore, we have

$$
\int_{0}^{\infty} \int_{0}^{L}\left|v_{t t}\right|^{2} \mathrm{~d} x \mathrm{~d} t \leq \int_{0}^{\infty} \int_{0}^{L}\left(7\left|A_{t t}\right|^{2}+28\left|\bar{u}_{0}\right|^{2}\left|A_{t x}\right|^{2}+7\left|\bar{u}_{0}\right|^{4}\left|A_{x x}\right|^{2}\right) \mathrm{d} x \mathrm{~d} t
$$




$$
\begin{aligned}
& +7\left|\bar{u}_{0}\right|^{2}\left(\frac{\pi}{L}\left|\bar{u}_{0}\right|+\eta\right)^{2} \int_{0}^{\infty} \int_{0}^{L}\left|A_{x}\right|^{2}+7 \zeta_{1}^{2} \int_{0}^{\infty} \int_{0}^{L} e^{-2 \eta t}|f(x, t)|^{2} \mathrm{~d} x \mathrm{~d} t \\
& +7 \zeta_{2}^{2} \int_{0}^{\infty} \int_{0}^{L}\left|v_{t}\right|^{2} \mathrm{~d} x \mathrm{~d} t+7 \zeta_{3}^{2} \int_{0}^{\infty} \int_{0}^{L}|\widetilde{v}|^{2} \mathrm{~d} x \mathrm{~d} t .
\end{aligned}
$$

Hence, we use (A.19), (A.17), (A.9), (A.8), (B.27), (B.29), and (B.19) to show that

$$
\int_{0}^{\infty} \int_{0}^{L}\left|v_{t t}\right|^{2} \mathrm{~d} x \mathrm{~d} t<\infty
$$

Similarly, one can show that $u_{x}, u_{y}, u_{x y}, u_{x x}, u_{y y}, v_{x} v_{y}, v_{x x}, v_{x t} v_{y t}, v_{y x}$, and $v_{y y}$ are in $L^{2}([0, L] \times(0, \infty))$, and these proofs are immediate.

\section{REFERENCES}

[1] B. Appelbe, D. Flynn, H. McNamara, P. O'Kane, A. Pimenov, A. Pokrovskii, D. Rachinskii and A. Zhezherun, Rateindependent hysteresis in terrestrial hydrology. IEEE Control Syst. Mag. 29 (2009) 44-69.

[2] B. Athukorallage and R. Iyer, Energy dissipation due to viscosity during deformation of a capillary surface subject to contact angle hysteresis. Physica B 435 (2014) 28-30.

[3] B. Athukorallage and R. Iyer, Investigation of energy dissipation due to contact angle hysteresis in capillary effect. J. Phys.: Conf. Ser. 727 (2016) 012003.

[4] B. Athukorallage, E. Aulisa, R. Iyer and L. Zhang, Macroscopic theory for capillary pressure hysteresis. Langmuir 31 (2015) 2390-2397.

[5] H. Chen, A. Amirfazli and T. Tang, Modeling liquid bridge between surfaces with contact angle hysteresis. Langmuir 29 (2013) 3310-3319.

[6] L. Cummings, S. Howison and J. King, Two-dimensional stokes and Hele-Shaw flows with free surfaces. Eur. J. Appl. Math. 10 (1999) 635-680.

[7] P.G. de Gennes, F. Brochard-Wyart and D. Quere, Capillarity and Wetting Phenomena: Drops, Bubbles, Pearls, Waves. Springer (2003).

[8] W. Deen, Analysis of Transport Phenomena. Oxford University Press (1998).

[9] C. Extrand and Y. Kumagai, An experimental study of contact angle hysteresis. J. Colloid Interface Sci. 191 (1997) 378-383.

[10] R. Finn, Equilibrium capillary surfaces. Springer-Verlag (1986).

[11] L. Gao and T.J. McCarthy, Contact angle hysteresis explained. Langmuir 22 (2006) 6234-6237.

[12] I. Gelfand and S. Fomin, Calculus of Variations. Dover Publications (2000).

[13] R. Holsapple, R. Venkataraman and D. Doman, New, fast numerical method for solving two-point boundary-value problems. J. Guidance Control Dyn. 27 (2004) 301-304.

[14] R. Iorio and V. Iorio, Fourier analysis and partial differential equations. Cambridge studies in advanced mathematics. Cambridge University Press (2001).

[15] H. Khalil, Nonlinear Systems, Prentice Hall PTR (2002).

[16] R. Ledesma-Aguilar, A. Hernández-Machado and I. Pagonabarraga, Three-dimensional aspects of fluid flows in channels. I. Meniscus and thin film regimes. Phys. Fluids 19 (2007) 102112.

[17] W. Rugh, Linear System Theory, Prentice-Hall information and systems sciences series. Prentice Hall (1993).

[18] F.S. Sherman, Viscous flow, McGraw-Hill series in mechanical engineering, McGraw-Hill (1990).

[19] J. Stoer and R. Bulirsch, Introduction to Numerical Analysis. Springer, New York, 2 ed. (2002).

[20] T.I. Vogel, Stability of a liquid drop trapped between two parallel planes. SIAM J. Appl. Math. 47 (1987) 516-525.

[21] D. White and J. Tallmadge, Theory of drag out of liquids on flat plates. Chem. Eng. Sci. 20 (1965) 33-37.

[22] P. Yan and A. Kassim, Mra image segmentation with capillary active contour, in Proceedings of the 8th international conference on Medical Image Computing and Computer-Assisted Intervention - Volume Part I, MICCAI'05, Berlin, Heidelberg. SpringerVerlag (2005) 51-58. 